\title{
Heritability of hippocampal functional and microstructural organisation
}

Şeyma Bayrak $(1,2,3) *$, Reinder Vos de Wael (4)*, H. Lina Schaare (1, 2), Benoit Caldairou (5), Andrea Bernasconi (5), Neda Bernasconi (5), Boris C. Bernhardt (4)*, Sofie L. Valk $(1,2)^{*}$

(1) Otto Hahn Group Cognitive Neurogenetics, Max Planck Institute for Human Cognitive and Brain Sciences, Leipzig 04103, Germany

(2) Institute of Neuroscience and Medicine, Research Center Jülich, Jülich 52428, Germany

(3) Department of Cognitive Neurology, University Hospital Leipzig and Faculty of Medicine, University of Leipzig, Leipzig 04103, Germany

(4) Multimodal Imaging and Connectome Analysis Laboratory, McConnell Brain Imaging Centre, Montreal Neurological Institute, McGill University, Montreal, QC H3A 2B4, Canada (5) Neuroimaging of Epilepsy Laboratory, McConnell Brain Imaging Centre, Montreal Neurological Institute, McGill University, Montreal, QC H3A 2B4, Canada

Correspondence to Şeyma Bayrak and Dr. Sofie L. Valk

Email: bayrak@.cbs.mpg.de, valk@.cbs.mpg.de

Keywords: hippocampus, hippocampal subfields, gradients, heritability, microstructure, functional connectivity

* These authors contributed equally. 


\section{Abstract}

The hippocampal formation is a uniquely infolded anatomical structure in the medial temporal lobe, involved in a broad range of cognitive and emotional processes. It consists of microstructually and functionally different subregions, such as subiculum (SUB), cornu ammonis (CA), and dentate gyrus (DG). Here we studied the heritability of hippocampal subfield structure and function, to dissociate genetic and environmental influences. Leveraging the twin set-up of the Human Connectome Project with multimodal neuroimaging, we show that both the functional connectivity and microstructure of hippocampal subfields were heritable. Notably, we observed a genetic relationship between hippocampal and isocortical microstructural organisation, suggesting that their microstructure is governed by shared genetic factors. Furthermore, combining structural and functional measures, we identified anterior-to-posterior and medial-to-lateral axes. Whereas posterior regions showed similar associations with isocortex between structure and function, anterior regions showed uncoupling of both metrics. Together, our findings illustrate the genetic basis of hippocampal structural and functional organisation, and suggest an uncoupling of both along their shared axes.

\section{Significance Statement}

The hippocampus is a key structure in cognition and affect, and a region that is highly susceptible to pathology in common neurological and neuropsychiatric conditions. Increasing evidence suggests that the hippocampus is not a uniform structure, but one that is composed of different subregions with specific microstructural and functional properties. Here, we studied how far these patterns are heritable, in order to dissociate genetic vs environmental effects on hippocampal microstructure and function. We could show the heritability of major structural and functional axes in the hippocampus which were found to be genetically coupled with the rest of the cortex. Moreover, we find that, across subfields, anterior portions show uncoupling between structure and function. Our study provides important insights on the heritability of functional and structural axes in the hippocampal formation and how these axes may scaffold flexible adaptation to a changing environment. 


\section{Introduction}

The hippocampal formation is a heavily infolded region in the medial temporal lobe and involved in a broad range of cognitive functions such as episodic memory ${ }^{1-3}$ and spatial navigation ${ }^{4}$ but also affective processes related to emotional reactivity ${ }^{5}$ and stress resilience ${ }^{6-8}$. Moreover, the hippocampus is a key region implicated in learning and plasticity ${ }^{9}$, and thus can be considered a model system to study how genetic and environmental factors interact in shaping brain organisation.

A three layered allocortex, the hippocampal formation consists of multiple subfields, or zones, starting at the subiculum (SUB) and moving outward to the hippocampus proper; the cornu ammonis (CA), and dentate gyrus (DG) ${ }^{10-13}$. These subfields have unique microstructural properties ${ }^{12,13}$ and have been suggested to participate differently in hippocampal circuitry ${ }^{14}$. Consequently, their contributions to functional processes are likely distinct ${ }^{15-17}$. Also, anatomical hippocampal-cortical projections are extensive and they have different characteristics based on the position within the hippocampal formation ${ }^{18,19}$. Tracer studies in rodents have shown that the ventral hippocampus is anatomically connected to the olfactory regions, prefrontal cortex, and amygdala, while the dorsal hippocampus is connected to the retrosplenial cortex, mammillary bodies, and anterior thalamus ${ }^{20,21}$. This ventral-dorsal transition in rodents may relate to an anterior-posterior (A-P) axis in humans ${ }^{22,23}$. Conversely, hippocampal infolding aligns with a medial-lateral (M-L) axis followed by the subfields, suggesting another transitional axis driven by the intracortical microstructure ${ }^{13,24}$. Thus, the hippocampal formation features two major axes, one from anterior to posterior regions, and the other along its infolding from SUB via CA to DG.

In humans, the hippocampal organisational axes observed in the (molecular) structure of the hippocampus are paralleled by the functional organisation of the hippocampus, as measured in vivo using functional MRI ${ }^{25}$. The A-P separation, so called long axis specialisation, has also been observed in resting-state fMRI (rs-fMRI) functional connectomics ${ }^{26-28}$. A second axis referred to as the transverse or medio-lateral axis emphasizes connectome differences in medial and lateral hippocampal portions ${ }^{18,24,29,30}$. In recent years, several groups have used dimension reduction techniques, commonly known as gradients, to provide a parsimonious account of 
hippocampal functional connectivity ${ }^{31-33}$. Gradient techniques allow a continuous description of the functional connectome compared to discrete network representations of the brain ${ }^{34-36}$. Using these gradients, prior studies have found relationships of hippocampal functional connectivity with anatomical organisation and microstructure ${ }^{33}$, as well as performance on memory recollection ${ }^{32}$, and pattern separation tasks ${ }^{31}$.

The different hippocampal zones and organisational axes are governed by partly non-overlapping genetic factors. In parallel to observations in rodents it has been shown that the human hippocampus shows variations in gene expression as a function of the A-P axis ${ }^{37}$. This genetic gradient is related to different macroscale networks in the cortex, dissociating behavioral systems associated with action and spatial cognition on the one hand and social and emotion/motivation on the other. Paralleling observations of differential genetic expression, hippocampal subfield volumes have been shown to be significantly heritable ${ }^{38}$ and to have genetic overlap with the total brain volume ${ }^{39}$. Moreover, recent work has indicated that the different hippocampal subfields have partly non-overlapping genetic signatures ${ }^{40}$ providing further evidence for the complex molecular biology associated with the hippocampal formation. However, it is not known whether sub regional differences in its structure and function are heritable. Moreover, other lines of research have reported the hippocampal structure to be highly plastic and reactive to stress ${ }^{41,42}$, suggesting not only genetic but also environmental factors may shape hippocampal organisation.

The structural model ${ }^{43}$, states that regions with a similar cytoarchitecture are also connected, and links isocortical cytoarchitecture to regional variations in plasticity and stability. Recent work on isocortical structure-function coupling suggests that coupling shows progressive reductions along an axis from unimodal to transmodal regions ${ }^{44-47}$. Such uncoupling may enable more flexible computations enabling abstract human cognition ${ }^{48,49}$ and is paralleled by reductions in genetic control from unimodal to transmodal regions ${ }^{44}$. In the hippocampus, posterior sub portions have been reported to show a predominant association with unimodal regions in structure and function whereas anterior sub portions are linked to transmodal regions 29,37. It is thus possible that the coupling of hippocampal microstructure and function shows meaningful variation along its large-scale axes, and helps to further understand the interrelation 
between plasticity and stability, or genetic and environmental factors, within this allocortical structure.

The overall goal of the current study is to map the heritability of hippocampal microstructure and function, and to study their interrelations. These analyses will help to further understand the relationship between the genetic basis of hippocampal organisation and its flexible functional role and plasticity. First we leveraged a connectome gradient framework to represent the functional axes and local transitions of microstructural similarity using $\mathrm{T} 1 \mathrm{w} / \mathrm{T} 2 \mathrm{w}$ images and computed their heritability using the twin set-up of the HCP S900 ${ }^{50}$. This enabled us to evaluate whether individual variations in intrinsic function and structure along these axes are heritable, i.e. under genetic control. Then we probed whether heritability of hippocampal-to-cortical patterns was systematically organised, allowing us to examine the genetic basis of functional and structural links between hippocampal formation and cortex. Last, we interrogated the shared organisation and coupling of hippocampal structure and function to understand spatial co-variation of structure-function associations within the hippocampal formation. We performed extensive robustness analysis to assess the stability of our findings. 


\section{Results}

We studied the heritability of hippocampal functional organisation and microstructure using the twin design Human Connectome Project (HCP) S900 data release ${ }^{50}$. Hippocampal subfields subiculum (SUB), CA1-3 (CA), and CA4-DG (DG) were delineated automatically using the SurfPatch algorithm ${ }^{51}$. After the quality assessment, there remained $\mathrm{n}=709$ participants (395 women, mean $\pm \mathrm{SD}$ age $=28.7 \pm 3.7 \mathrm{y}, 176$ monozygotic twins, 178 siblings without twin status and 355 participants without familial relatedness) accessible for our study. To probe functional organisation, we first obtained the subfield-isocortical functional connectivity (FC) and then implemented an unsupervised dimension reduction technique on FC measures, yielding connectome gradients 33,52 (Fig. 1). Hippocampal microstructural features were addressed by projecting an in vivo proxy for myelination, the ratio of T1- over T2-weighted (T1w/T2w) image intensities ${ }^{53}$ (Fig. 2). Additionally, to assess the microstructural congruence between hippocampus and isocortex, we computed structural intensity covariance ( $\mathrm{SiC}$ ) of the T1w/T2w maps and the corresponding genetic correlation (Fig. 3). Finally, we explored whether functional and microstructural organisation together underlie a common hippocampal axis. To do so, we obtained their multimodal gradient representations by fusing both modalities ${ }^{54}$, and evaluated the association between hippocampal function and structure (Fig. 4). All heritability and genetic correlation analyses were performed using the Sequential Oligogenic Linkage Analysis Routines (SOLAR, v8.5.1) ${ }^{55}$. Details on the subject selection as well as the methods can be found in the SI Appendix.

\section{Hippocampal-isocortical functional organisation is marginally heritable.}

We first correlated the rs-fMRI time series of each subfield with the time series of isocortical parcels to compute the hippocampal-isocortical functional connectivity (FC) in our sample (Fig. 1A). To illustrate the spatial organisation of subfield-specific FC, we derived hippocampal connectivity gradients, consistent with Vos de Wael (2018) (Fig. 1B). Each connectivity gradient represented a particular axis of FC. Gradient $1\left(G 1_{F C}\right)$ presented an anterior-posterior (A-P) axis across hippocampal subfields and explained $24 \%$ of the variance, whereas Gradient $2\left(G 2_{F C}\right)$ described a medial-lateral (M-L) axis and explained $9 \%$ of the 
variance (Fig. S1B). The A-P transitional axis was observed consistently for all subfields. However, the gradient loadings of the M-L axis decreased from SUB to CA, and were minimal along the DG. To further quantify the regional associations between the hippocampal A-P axis and the whole brain connectome, we correlated the subfield $G 1_{F C}$ with the subfield-isocortical FC (Pearson's r, Fig. 1C). Anterior hippocampal portions (blue in Fig. 1B) were functionally coupled to sensorimotor, default-mode and limbic networks (blue in Fig. 1C, Fig. 1D) for SUB. However, the strength of these couplings decreased towards CA and DG. Posterior hippocampal portions (yellow in Fig. 1B) were functionally more connected to fronto-parietal, salience, dorsal attention and visual networks (yellow in Fig. 1C, Fig. 1D) for DG, with decreasing connectivity strength for CA and SUB.

The strongest hippocampal-isocortical functional couplings were found to be in the default-mode, somatomotor, visual and limbic areas (Fig. S2A, Fig. S2D). Next, we ran heritability analyses ${ }^{55}$ on these functional couplings to illustrate the impact of familial relatedness on the hippocampal-isocortical connectome (Fig. S2B). Heritability $\left(h^{2}\right)$ of SUB-isocortex FC was the highest in ventromedial, posterior cingulate, and precuneus areas, linked to sensorimotor, limbic, and default mode functional networks respectively. A similar heritability pattern was observed for CA-isocortex FC, with highest heritability in sensorimotor and salience networks. For the DG-isocortex FC, compared to SUB- and CA-isocortex FC, we observed higher heritability in primary sensorimotor areas and the superior temporal lobe, associated with sensorimotor and dorsal attention functional networks. The $h^{2}$ values were found to be significant throughout most of the cortical areas using a likelihood ratio test (Fig. S2C).

Following, we obtained the heritability of the A-P and M-L functional gradient axes, $h^{2}\left(G 1_{F C}\right)$ and $h^{2}\left(G 2_{F C}\right)$ respectively (Fig. 1E), to assess whether individual variations in local gradient loadings were heritable. For SUB and CA, $h^{2}\left(G 1_{F C}\right)$ was found to be modest (mean: 0.14, range: [0.06, 0.30] and mean: 0.13, range: [0, 0.26] respectively). However, it was low for DG (mean: 0.05 , range $[0,0.16])$. The second gradient's heritability, $h^{2}\left(G 2_{F C}\right)$, was found to be low for all subfields (SUB: mean: 0.05, range: [0, 0.18], CA: mean: 0.08, range: [0, 0.31], and 
DG: mean: 0.00, range: [0, 0.04]). The heritability was significant $(\mathrm{FDRq}<0.05)$ only for the anterior and posterior portions of the SUB- $G 1_{F C}$ and lateral portions of CA-G2 ${ }_{F C}$ (Fig. S2E). Finally, we evaluated whether the heritability of subfield-to-isocortical connectivity was systematically organised, by performing gradient decomposition on the heritability scores of the subfield functional connectome (Fig. 1F). The primary gradient of the FC heritability G1 $\left(h^{2}\right)$ did not reveal a consistent transition pattern along the subfields. However, the secondary gradient of the FC heritability G2 $\left(h^{2}\right)$ depicted an A-P separation of the $h^{2}$ profiles for SUB and DG. 
A Hippocampal subfield segmentations and functional connectivity analysis
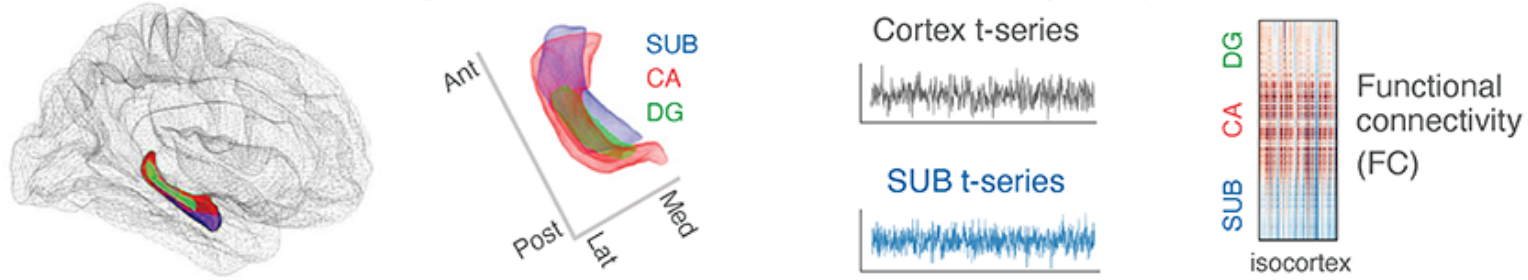

B Principal and secondary gradients of subfield functional connectivity
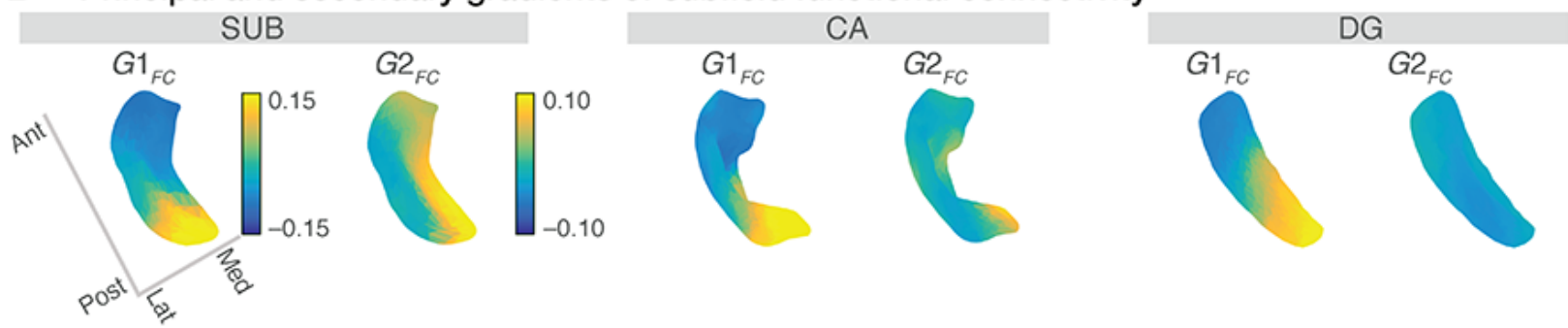

C Isocortical projections of anterior-posterior axis on the functional connectivity
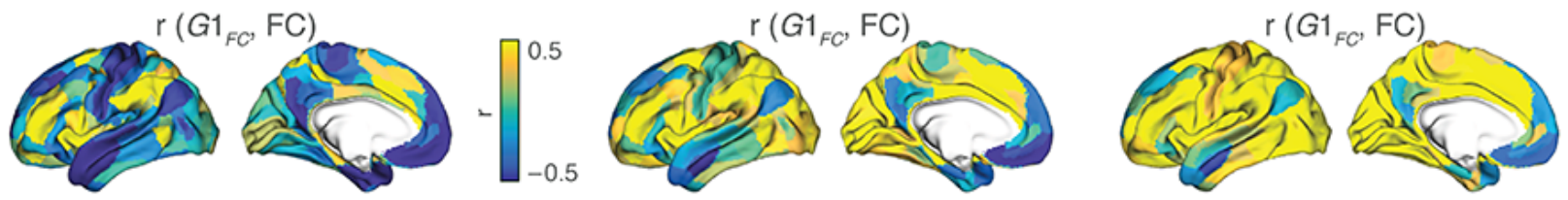

D Network based functional couplings of anterior-posterior axis

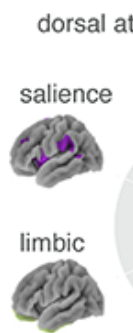

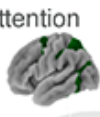

sensorimotor
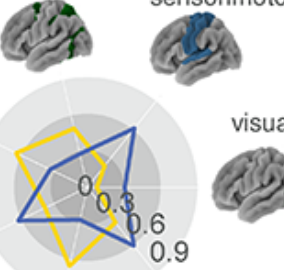

0.9

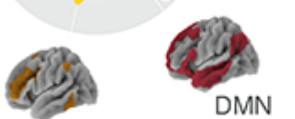

$\mathrm{DMN}$

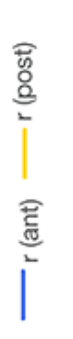

dorsal attention

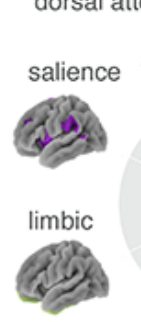

fronto-parietal

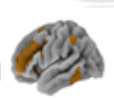

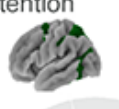

sensorimotor
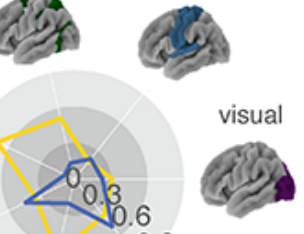

0.9

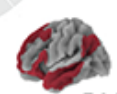

fronto-parietal

$\mathrm{DMN}$

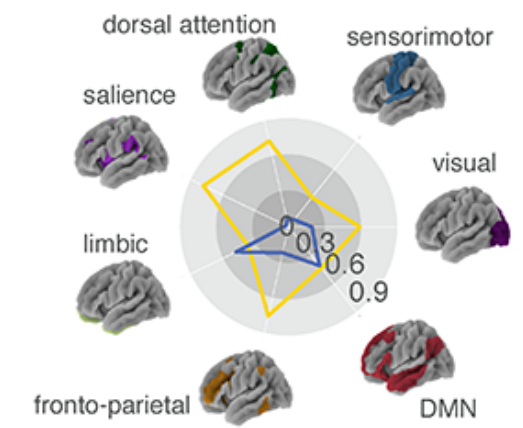

\section{E Heritability of functional connectivity gradients}
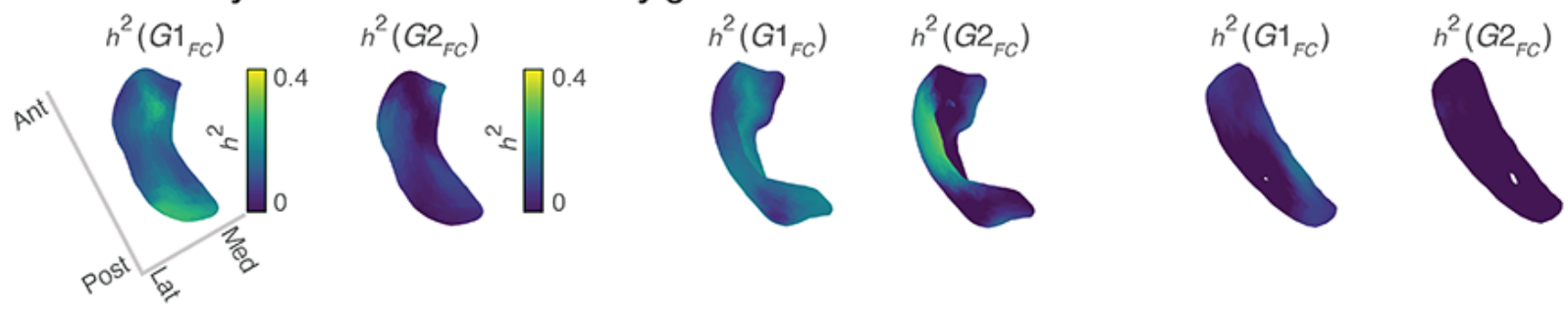

F Principal and secondary gradients of subfield connectivity heritability
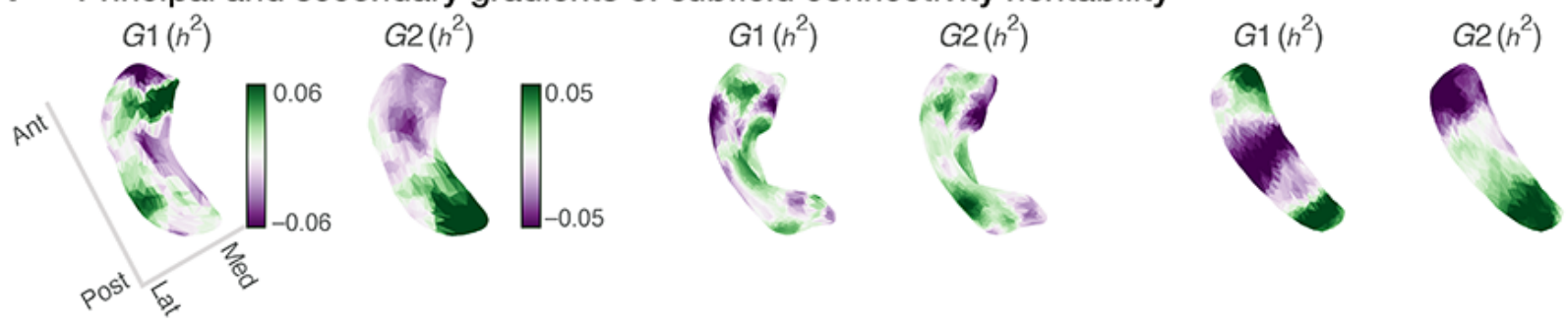
Fig. 1. Hippocampal-isocortical functional organisation and its heritability. A. Hippocampal subfield surfaces were automatically delineated using SurfPatch ${ }^{51}$ : subiculum (SUB, blue), CA1-3 (CA, red), and CA4-DG (DG, green). rs-fMRI time series were extracted along the individual subfields and correlated with the time series of the isocortex to obtain subfield-isocortex functional connectivity (FC). B. Connectivity gradients of subfield-isocortical FC for SUB (left), CA (middle) and DG (right). Gradient 1 $\left(G 1_{F C}\right)$ depicts an anterior-posterior connectivity axis, whereas Gradient 2 (G2 $F C$ displays a medial-lateral axis. C. Variations in hippocampal-isocortical FC across the $G 1_{F C}$ projected on the isocortex (Pearson's r-values). Lower r-values (blue) indicate FC similarity between the anterior subfield portions and isocortex, whereas higher r-values (yellow) that of the posterior subfield portions and isocortex. D. FC from anterior (r-values, blue) and posterior (r-values, yellow) subfield portions were distributed into large-scale functional networks. E. Heritability of subfield FC gradients $\left(h^{2}\left(G 1_{F C}\right)\right.$ and $h^{2}\left(G 2_{F C}\right)$ ). F. Gradients of hippocampal-isocortical mean FC heritability. Gradient 2 of the FC heritability (G2 $\left(h^{2}\right)$ ) depicted an anterior-posterior separation of the $h^{2}$ profiles for SUB and DG.

\section{Hippocampal subfield microstructure is highly heritable.}

To study the microstructural properties of the hippocampal subfields, we used T1w/T2w intensity maps. Mean T1w/T2w intensity profiles distributed along the subfields did not reveal any potential outliers (mean $\pm \mathrm{SD}=2.02 \pm 0.41$ for $\mathrm{SUB}, 2.01 \pm 0.78$ for $\mathrm{CA}$, and $1.66 \pm 0.22$ for DG) (Fig. 2A). To evaluate the topographical relationship between functional organisation and microstructural profiles, we correlated the secondary FC gradient $\left(G 2_{F C}\right)$ with the T1w/T2w maps for each subject $(\mathrm{n}=709)$ and subfield (Fig. 2B). Individual-level correlations were found to be significantly positive across participants for $\operatorname{SUB}(\bar{r}=0.53, p<0.005$, one-tailed Wilcoxon signed-rank test) and CA $(\bar{r}=0.20, p<0.005)$, however not for DG $(\bar{r}=-0.02, p=0.21)$. Our findings were consistent with the right hemispherical subfields for both at the individual and group level (Fig. S3C, Fig. S3D). These associations were heritable for CA but not for SUB and DG (SUB: $\left.h^{2}=0.09, p=0.1, \mathrm{CA}: h^{2}=0.13, p=0.03, \mathrm{DG}: h^{2}=0.00, p>0.1\right)$.

To evaluate the spatial similarity between local microstructure and functional gradients, we quantified the group-level association between the T1w/T2w and $G 2_{F C}$ for subfields (Pearson's r) and its significance level with the variogram approach $\left(p_{\text {vario }}\right)^{56}$ that controls for the spatial autocorrelations (Fig. 2C). $G 2_{F C}$ had the strongest association with the $\mathrm{T} 1 \mathrm{w} / \mathrm{T} 2 \mathrm{w}$ profiles for 
SUB $\left(r=0.93, p_{\text {vario }}<0.001\right)$ and less with the other subfields $\left(\mathrm{CA}: \mathrm{r}=0.23, p_{\text {vario }}=0.02\right.$, DG: $\mathrm{r}$ $\left.=-0.01, p_{\text {vario }}=0.9\right)$. After correcting for the rs-fMRI temporal signal-to-noise ratio, the associations between $\mathrm{T} 1 \mathrm{w} / \mathrm{T} 2 \mathrm{w}$ profiles and $G 2_{F C}$ maps were preserved. Finally, we aimed to evaluate whether the spatial variation of microstructure along the hippocampal subfields itself was heritable by computing heritability for the $\mathrm{T} 1 \mathrm{w} / \mathrm{T} 2 \mathrm{w}$ intensity profile maps (Fig. 2D). T1w/T2w maps were highly heritable across all subfields, reaching up to $h^{2}=0.77$ for SUB (mean $\pm \mathrm{SD}=0.44 \pm 0.15$ for $\mathrm{SUB}, 0.41 \pm 0.13$ for $\mathrm{CA}$, and $0.43 \pm 0.07$ for $\mathrm{DG}$ ). Multiple comparison corrections using FDR reported significant heritability scores across almost all subfield vertices (100\% of SUB vertices, $99 \%$ of CA and DG vertices). By adjusting for mean $\mathrm{T} 1 \mathrm{w} / \mathrm{T} 2 \mathrm{w}$ as a covariate in the heritability model, we found similar heritability patterns for individual subfields, and both hemispheres (Fig. S4). This indicated that the heritability of subfields was present beyond any mean $\mathrm{T} 1 \mathrm{w} / \mathrm{T} 2 \mathrm{w}$ intensity variation across individuals. 
A Individual $(n=709)$ mean $T 1 w / T 2 w$ intensity

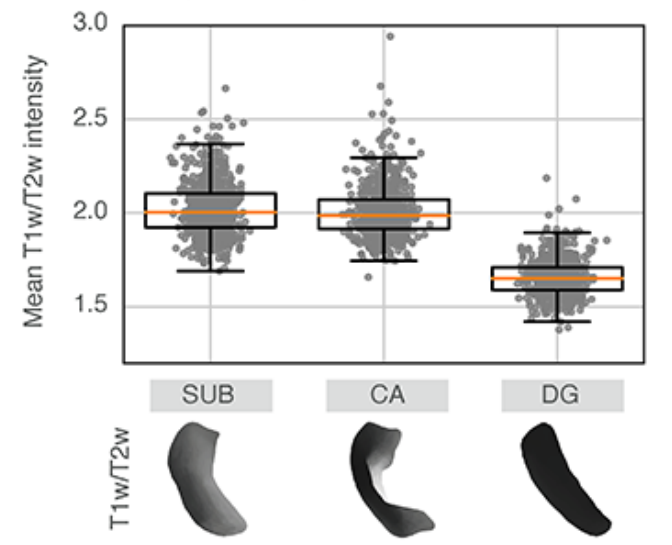

B Individual correlations of $\mathrm{T} 1 \mathrm{w} / \mathrm{T} 2 \mathrm{w}$ and $\mathrm{G} 2_{\mathrm{FC}}$

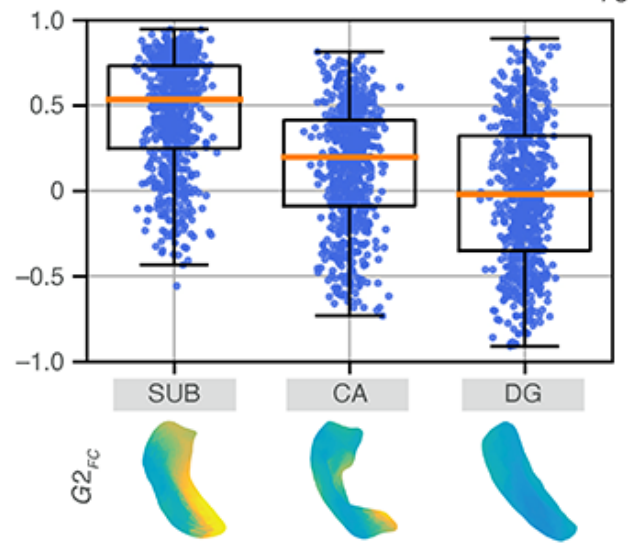

C Group-level association between $\mathrm{T} 1 \mathrm{w} / \mathrm{T} 2 \mathrm{w}$ intensity maps and $\mathrm{G} 2_{F C}$
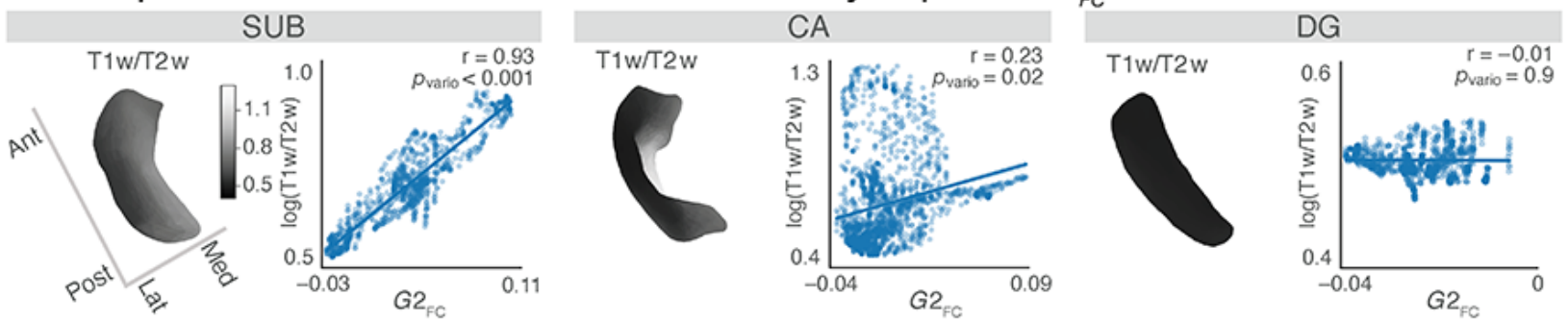

D Heritability of T1w/T2w intensity maps
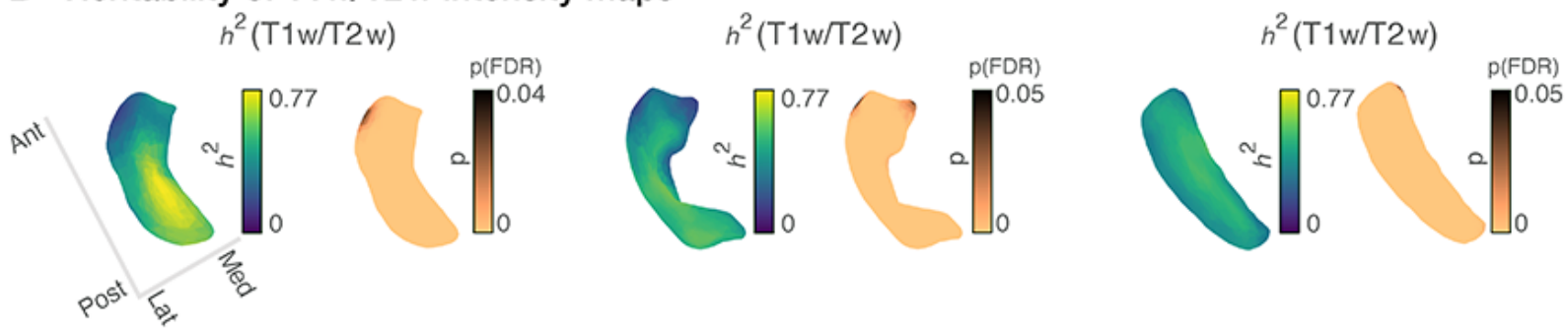

Fig. 2. Subfield T1w/T2w intensity maps, its association with the secondary functional connectivity gradient, and its heritability A. Mean $\mathrm{T} 1 \mathrm{w} / \mathrm{T} 2 \mathrm{w}$ intensity profile distributions are demonstrated across participants $(\mathrm{n}=709)$ and for each subfield (SUB, CA, and DG). Mean and standard deviations of T1w/T2w maps were $2.02 \pm 0.41$ for SUB, $2.01 \pm 0.78$ for CA, and $1.66 \pm 0.22$ for DG. B. Individual correlations $(\mathrm{n}=709)$ between $G 2_{F C}$ maps and $\mathrm{T} 1 \mathrm{w} / \mathrm{T} 2 \mathrm{w}$ intensity maps for each subfield. Individual correlations $\left(\mathrm{r}\left(G 2_{F C}, \log (\mathrm{T} 1 \mathrm{w} / \mathrm{T} 2 \mathrm{w})\right)\right.$ were significantly positive for the $\mathrm{SUB}$ (median $\bar{r}=0.53, p<0.005$, one-tailed Wilcoxon signed-rank test $)$ and CA $(\bar{r}=0.20, p<0.005)$, however not for DG $(\bar{r}=-0.02, p=$ $0.2)$. C. Group-level association between the mean $\mathrm{T} 1 \mathrm{w} / \mathrm{T} 2 \mathrm{w}$ profiles and the $G 2_{F C}$ for each subfield. The $\mathrm{T} 1 \mathrm{w} / \mathrm{T} 2 \mathrm{w}$ profiles of subfields correlate strongly with the $G 2_{F C}$ for SUB $\left(\mathrm{r}=0.93\right.$ and $\left.p_{\text {vario }}<0.001\right)$, however, not for the CA $\left(\mathrm{r}=0.23\right.$ and $\left.p_{\text {vario }}=0.02\right)$ or $\mathrm{DG}\left(\mathrm{r}=-0.01\right.$ and $\left.p_{\text {vario }}=0.9\right)$. D. Heritability of subfield $\mathrm{T} 1 \mathrm{w} / \mathrm{T} 2 \mathrm{w}$ profiles $\left(h^{2}(\mathrm{~T} 1 \mathrm{w} / \mathrm{T} 2 \mathrm{w})\right)$ and its significance levels. T1w/T2w maps were strongly heritable across all subfields. p-values were reported after multiple comparison corrections using FDR (copper color denotes pFDR $<0.05$, black pFDR $>0.05$ ). 


\section{Hippocampal-isocortical structural intensity covariance is genetically correlated.}

Having shown that: 1) FC organisation of hippocampal subfields is marginally heritable and 2) microstructural organisation of hippocampal subfields is highly heritable, we explored whether the structural association between hippocampus and isocortex was related with shared genetic factors. To uncover the microstructural similarity between hippocampal subfields and isocortex, we first computed the structural intensity covariance ( $\mathrm{SiC}$ ) of $\mathrm{T} 1 \mathrm{w} / \mathrm{T} 2 \mathrm{w}$ maps across all participants $(n=709)$ between subfields and isocortex. We then evaluated its low-dimensional representations by means of dimension reduction ${ }^{57}$ (Fig. 3A). The principal gradient of SiC ( $\left.G 1_{S i C}\right)$ revealed an A-P organisational axis across all the subfields (Fig. 3B). Further, we observed a high similarity between $G 1_{S i C}$ and $G 1_{F C}$ (SUB: Pearson's $\mathrm{r}=0.88, \mathrm{CA}: \mathrm{r}=0.86$, and DG: $r=0.88, p_{\text {vario }}<0.001$ for all subfields). However, the second gradient of $\operatorname{SiC}\left(G 2_{S i C}\right)$ did nor represent a converging organisational pattern for the subfields, rather $G 2_{S i C}$ reflected the local subfield transitions as a whole for SUB, with an M-L transitional axis for CA and DG. Similarities between $G 2_{S i C}$ and $G 2_{F C}$ were reported to be weaker compared to the primary gradients (SUB: $\mathrm{r}=0.36, p_{\text {vario }}=0.015, \mathrm{CA}: \mathrm{r}=0.22, p_{\text {vario }}=0.015$, and $\mathrm{DG}: \mathrm{r}=-0.15, p_{\text {vario }}=$ 0.2). Evaluating the pattern of correlation between subfield-isocortex $\mathrm{SiC}$ and $G 1_{S i C}$, we could assess how hippocampal and isocortical regions spatially relate to each other in terms of their microstructural similarity (Fig. 3C). Anterior hippocampal portions (blue in Fig. 3B) shared more microstructural similarity with the anterior isocortex, while the posterior hippocampal portions (yellow in Fig. 3B) were more related to the posterior isocortex, particularly for SUB and CA. For the DG we observed less divergent patterns of subfield-isocortical similarity between its anterior and posterior portions.

Next, we assessed the genetic similarity of hippocampal and isocortical SiC. To do so we computed the genetic correlations (GEN) of SiC and then decomposed the GEN on its gradients (Fig. 3D). The principal gradient of the GEN $\left(G 1_{G E N}\right)$ displayed an A-P axis for all the subfields. Similarities between $G 1_{G E N}$ and $G 1_{F C}$ were lower (SUB: r = 0.67, CA: r = 0.41, DG: $\mathrm{r}=0.75$, and $p_{\text {vario }}<0.001$ for all subfields) compared to similarities between $G 1_{S i C}$ and $G 1_{F C}$. The second 
gradient of GEN $\left(G 2_{G E N}\right)$ did not reveal a consistent organisational axis for SUB, however, we observed an M-L axis for $\mathrm{CA}$ and DG. Analogous to $\mathrm{SiC}$, we also investigated the correlation between hippocampal-isocortical GEN variations and $G 1_{G E N}$ (Fig. 3E). Indeed, patterns were largely mirroring those observed in $\mathrm{SiC}$, indicating that the structural intensity covariance between hippocampus and isocortex is largely concordant with genetic patterning. Whereas posterior regions were associated with unimodal regions for SUB, anterior subfield regions showed a link to, in particular frontal, transmodal, areas. 
A Hippocampal-isocortical structural intensity covariance and genetic correlations
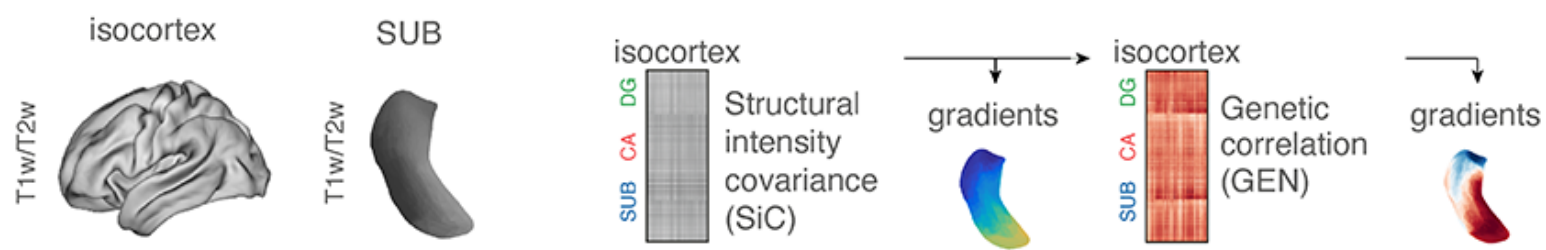

B Principal and secondary gradients of subfield structural intensity covariance SUB CA
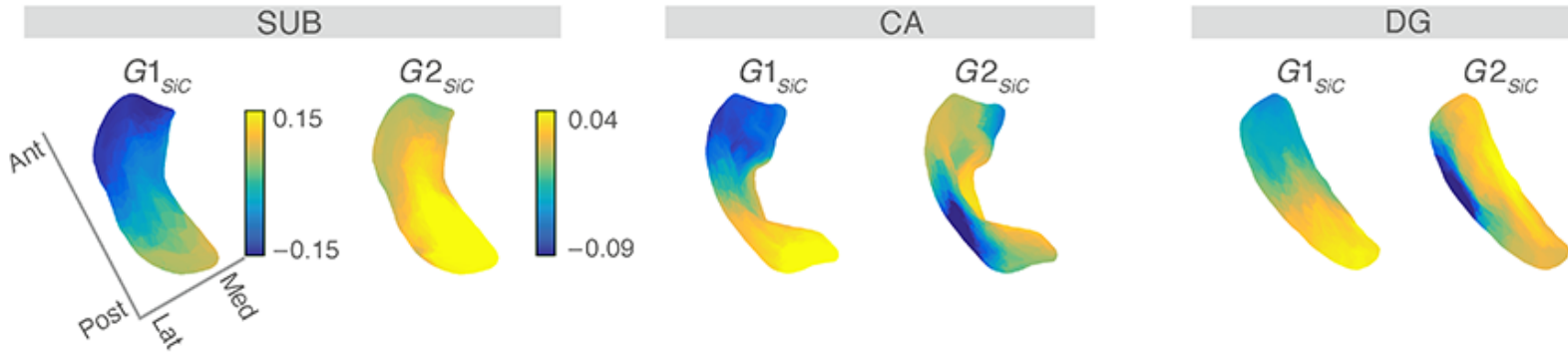

C Isocortical projections of anterior-posterior axis on structural intensity covariance
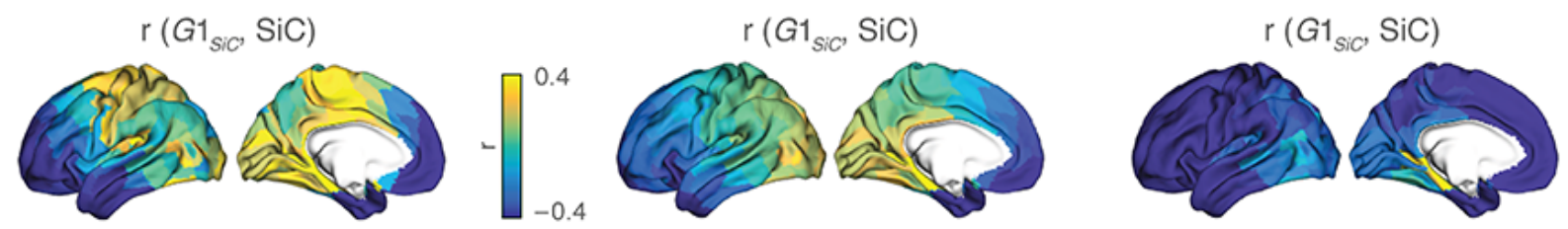

D Principal and secondary gradients of subfield genetic correlations
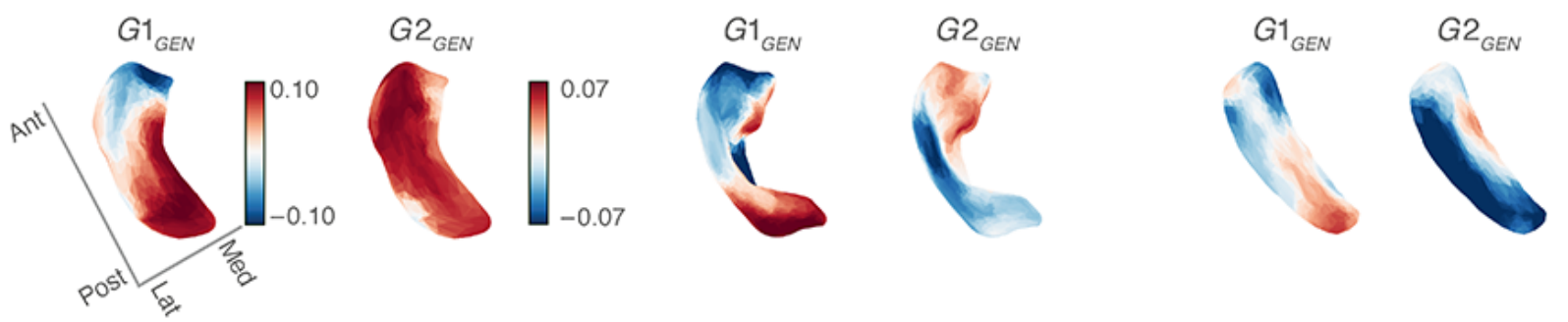

E Isocortical projections of anterior-posterior axis on the genetic correlations
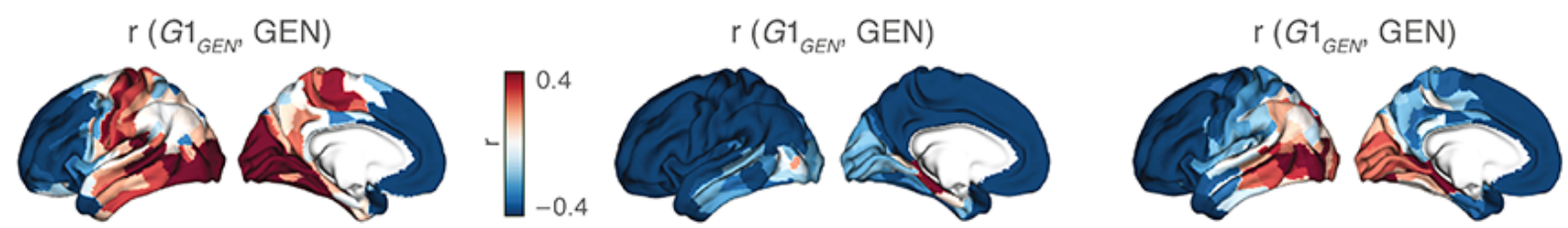

Fig. 3. Organisation of hippocampal-isocortical structural intensity covariance ( $\mathrm{SiC}$ ) and its genetic correlations (GEN). A. Hippocampal-isocortical SiC was assessed by correlating hippocampal and isocortical $\mathrm{T} 1 \mathrm{w} / \mathrm{T} 2 \mathrm{w}$ intensity maps across participants and subfields. Shared genetic variations in $\mathrm{T} 1 \mathrm{w} / \mathrm{T} 2 \mathrm{w}$ intensity maps were assessed by conducting a genetic correlation analysis on the SiC. Both $\mathrm{SiC}$ and GEN matrices were then decomposed into their gradient representations, separately. B. Gradients of SiC for SUB (left), CA (middle), and DG (right). $G 1_{S i C}$ represents an anterior-posterior (A-P) axis for all subfields, whereas $G 2_{S i C}$ reflects the differential axis of local transitions for individual subfields. $\mathbf{C}$. Variations in $\mathrm{SiC}$ across its $G 1_{S i C}$ projected on the isocortex (Pearson's r-values). Lower r-values (blue) 
indicate $\mathrm{SiC}$ similarity between the anterior subfield portions and isocortex, whereas higher r-values (yellow) that of the posterior subfield portions and isocortex. D. Gradients of GEN for SUB (left), CA (middle), and DG (right). $G 1_{G E N}$ represents an A-P axis for all subfields, whereas $G 2_{G E N}$ reflects the differential axis of local transitions for individual subfields. E. Variations in GEN across its $G 1_{G E N}$ projected on the isocortex (Pearson's r-values). Lower r-values (dark blue) depict shared genetic influence between anterior subfield portions and isocortex and higher r-values (red) that of posterior subfield portions and isocortex.

Fused gradients of hippocampal-isocortical functional and structure reveal the anterior-posterior axis.

Last, we explored concordances in spatial variation of structure-function associations within the hippocampal formation. To do so, we obtained the fused gradients by decomposing FC and SiC measures simultaneously (Fig. 4, upper panels). The principal fusion gradient $G 1_{f u s}$ emphasized anteromedial hippocampal portions for SUB and mediolateral portions for CA and $\mathrm{DG}$, and show most resemblance to the patterns observed in $G 2_{F C}\left(\mathrm{SUB}: \mathrm{r}=0.46, p_{\text {vario }}<0.001\right.$, $\left.\mathrm{CA}: \mathrm{r}=0.14, p_{\text {vario }}=0.11, \mathrm{DG}: \mathrm{r}=0.22, p_{\text {vario }}=0.03\right)$ and local T1w/T2w (SUB: $\mathrm{r}=0.47, \mathrm{CA}$ : $\mathrm{r}=0.52$, DG: 0.39). The second fused gradient $G 2_{f u s}$ revealed an A-P organisational axis for all subfields, which shared high similarity with $G 1_{F C}$ and $G 1_{S i C} \cdot\left(G 2_{f u s}\right.$ and $G 1_{F C}:$ SUB: $\mathrm{r}=0.89$, CA: $r=0.89$, DG: $r=0.86$ and $p_{\text {vario }}<0.001 . G 2_{f u s}$ and $G 1_{S i C}:$ SUB: $r=0.73, \mathrm{CA}: \mathrm{r}=0.83$, DG: $\mathrm{r}=0.80$ and $p_{\text {vario }}<0.001$ for all) (Table S1). Both fused gradients showed distinct functional associations with the isocortex and similar to our observations for $G 1_{F C}$. $G 2_{f u s}$ dissociated default as well as sensory-motor networks from attention and visual networks for respectively anterior and posterior portions of the gradient (Fig. S5B, S5C). This dissociation mirrored the spatial distribution of the tertiary large scale rs-FC gradients ${ }^{36}$ (Fig. S6) underscoring the link between hippocampal and cortical organisation.

Last, we evaluated structural and functional subfield-to-isocortical patterning. Thus, we obtained the coupling maps of the FC and SiC measures by correlating the two measures at the subfield vertex-level (Fig. 4, upper panels). Higher coupling values indicate a spatial agreement between functional and microstructural isocortical profiles for the subfield vertices, whereas 
lower values denote a disagreement - an uncoupling. The mutual subfield vertices with the highest coupling values were observed to be located at the lateral/posterior portions of the SUB, $\mathrm{CA}$ and DG, whereas low coupling values were located at anterior/medial portions of the subfields. To synthesize the coupling of structure and function in the hippocampus with structural and functional organisational axes, associations between all axes were quantified by the coefficient of determination $\left(R^{2}\right)$ (Fig. 4, lower panels). Coupling is aligned in particular to anterior-to-posterior axes for the subfields CA and DG, and medial-to-lateral axes for the SUB. This observation highlights the unique distribution of structure and function in hippocampal subfields, suggesting uncoupling of structure and function along its organisational axes. 
bioRxiv preprint doi: https://doi.org/10.1101/2021.11.10.468049; this version posted January 27, 2022. The copyright holder for this preprint (which was not certified by peer review) is the author/funder. All rights reserved. No reuse allowed without permission.
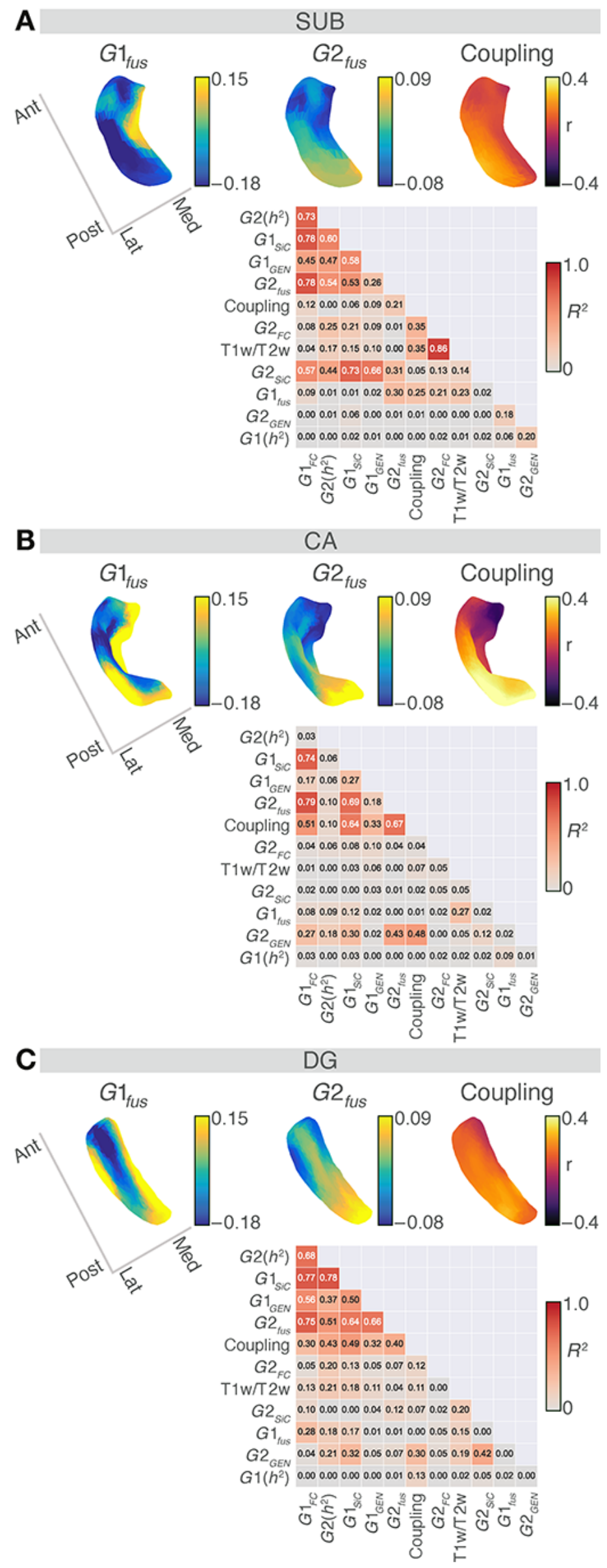
Fig. 4. Hippocampal fused gradients, coupling maps and associations among hippocampal organisational axes for individual subfields. A. SUB: Primary and secondary fused gradients, $G 1_{f u s}$ and $G_{\text {fus }}$, and the vertex-wise coupling map between hippocampal-isocortical functional connectivity (FC) and structural intensity covariance ( $\mathrm{SiC}$ ) measures (upper panel). $G 1_{\text {fus }}$ separates the anterior-medial portions (yellow) from the posterior-lateral portions (blue). G2 fus displays an anterior-posterior (A-P) axis. Higher coupling values (r) denote an association between FC and SiC, whereas lower coupling values display a dissociation between them. The coefficient of determination $R^{2}$ computed for organisational subfield axes in all possible pairwise combinations (lower panel). High $R^{2}$ values (red) indicate a strong spatial alignment between the organisational axes, whereas low $R^{2}$ values (light gray) an unalignment. B. CA: $G 1_{\text {fus }}$ separates anterior-medial and posterior-lateral portions (yellow) from the remaining subfield portions (blue). Both the $G 2_{f u s}$ and the coupling map display an A-P axis. C. DG: $G 1_{f u s}$ separates medial-lateral portions (yellow) from the remaining subfield portions (blue). Both the $G 2_{f u s}$ and the coupling map display an A-P axis. 


\section{Discussion}

The hippocampus is an anatomically unique structure that is considered to be a hub for cognitive and emotional processes. Here, we studied the functional and structural organisation of the hippocampal formation and explored its genetic association with the isocortex through heritability analyses. Building on emerging work describing the coalescence of anterior-to-posterior and medial-to-lateral gradients of microstructure and function in the hippocampal formation in vivo ${ }^{18,29,33,58}$, we describe how structure and function are co-organised in the hippocampal formation. First, we found that functional organisation of hippocampal subfields was marginally heritable. Spatial variations in subfield T1w/T2w intensity maps, serving as a marker for myelin-related microstructure, were heritable and genetically related to functional organisation. Moreover, we showed that structural intensity covariance of T1w/T2w between hippocampal subfields and isocortex followed an anterior-posterior (A-P) and a medial-lateral (M-L) axis. These patterns were genetically correlated, indicating that surface structure of the subfields underlie shared genetic influences with the isocortex. Last, integrating structural and functional metrics in a unified framework, we observed evidence for shared M-L and A-P axes in both metrics, further corroborating the notion of these two axes of structural and functional organisation within the hippocampal formation. However, evaluating the similarity of structure-function coupling in the hippocampal subfields with the cortex, we found in particular lateral/posterior regions to be highly coupled, whereas anterior/medial regions were uncoupled. Whereas posterior subfield portions showed structural and functional associations with posterior and unimodal cortical networks, anterior subfield regions were linked to anterior and transmodal cortical networks, including the frontal cortex.

To study the subregional organisation of the hippocampal formation, we automatically segmented the hippocampal formation via a subfield and surface-based approach (SUB, CA, and DG) ${ }^{51}$, which has been previously validated in both healthy individuals and those with hippocampal pathology ${ }^{59}$. Such surface-based approaches improve anatomical alignment across individuals ${ }^{58}$. Previously, animal studies have delineated the hippocampus into different zones based on its cytoarchitecture and attributed specific connectivity features to these zones ${ }^{13}$. Similar findings were reported in humans based on neurotransmitter distributions ${ }^{10}$ and ex-vivo 
MRI ${ }^{29}$. In the current work, we observed varying hippocampal-cortical functional connectivity (FC) profiles across subfields. The primary gradient $G 1_{F C}$ demonstrated A-P transitions (long axis ${ }^{19,26-28}$ ), whereas the secondary gradient $G 2_{F C}$ revealed M-L separations (transverse axis ${ }^{18,24,30}$ ). The A-P axis was equally predominant in all subfields. However, the M-L axis was the most distinct for the SUB. Further bridging the M-L axis and T1w/T2w maps, the M-L axis was found to align strongly with the microstructural proxy, particularly for the SUB and to a lesser extent in CA. In sum, the long axis specialisation was preserved in all subfields, whereas the transverse axis indicated a link between intrinsic FC and microstructure, particularly in SUB.

Functional connectivity and structural organisation of the hippocampal subfields was heritable, indicating individual variation was partly attributable to genetic factors. Hippocampal-isocortical FC was moderately heritable and its strength increased in the sensorimotor and dorsal attention domains from the SUB to CA and finally to DG. Studying the heritability of structural and functional organisation within subfields we found that heritability estimates were highest within the subfield microstructure proxy (T1w/T2w) and lowest for the A-P $\left(G 1_{F C}\right)$ and M-L $\left(G 2_{F C}\right)$ functional hippocampal gradients. Indeed, the heritability of $G 1_{F C}$ and $G 2_{F C}$ itself was not significant, indicating that individual variation within functional gradients did not vary as a function of genetic proximity of individuals. At the same time we found that heritability of subfield-isocortical functional connectivity was again organised along an A-P axis, indicating that anterior and posterior portions of hippocampal subfields have distinct heritable relations with the isocortex. The $\mathrm{T} 1 \mathrm{w} / \mathrm{T} 2 \mathrm{w}$ microstructure along the hippocampal subfield surfaces was highly heritable, and showed genetic correlation with M-L gradient in function. Previously, volume-based studies segmented the hippocampus into 12 subregions and showed that these volumes exhibit strong heritability ${ }^{38,60}$. Here, we extended this work by studying the heritability of subtle variations of structure and function within subfield surfaces, as well as their link to the isocortex. Moreover, genome-wide studies identified single-nucleotide polymorphisms (SNP) associated with hippocampal volumes ${ }^{61-63}$ showing, in part, unique SNPs for each subfield ${ }^{64}$. Quantifying the degree of heritability of the functional or structural properties could elevate our knowledge around the role of genetic constraints and environmental factors shaping the hippocampal formation. For instance, environmental influences on brain 
plasticity can be interpreted as a degree of aberration from the heritability of rs-fMRI connectivity, i.e. the less heritable the $\mathrm{FC}$ of a brain region, the larger the potential environmental influence ${ }^{44,65,66}$. It is thus possible that the low heritability of functional organisation of subfields reflects these variations, attributable to environmental effects and associated hippocampal plasticity. Indeed, previous work in rodents and human adults has shown high plasticity of the hippocampus in both species ${ }^{67}$, which has been linked to internal and external changes, such as hormonal levels and stress responses ${ }^{42}$.

Evaluating the structural association between hippocampal subfields and isocortex we observed that axes of microstructure within each subfield had unique genetic correlations with the isocortex, obtained by probing genetic correlation of structural intensity covariance (SiC) measures between the subfields and isocortex. Overall, $\mathrm{SiC}$ emphasizes the morphological similarity among brain regions ${ }^{68}$. Although the decomposed $\mathrm{SiC}$ measure originated from the T1w/T2w maps, its low dimensional components depicted similar spatial organisation to that of the functional maps. The primary genetic gradient $\left(G 1_{G E N}\right)$ revealed an A-P axis for all the subfields, whereas the secondary gradient $\left(G 2_{G E N}\right)$ revealed an M-L axis for CA and DG. In general, the A-P genetic axis of SUB and DG was related to an A-P separation along the cortex, whereas it was bounded within the hippocampal region for the CA. Earlier studies have presented an isocortex-wide A-P topography derived from cortical thickness morphology ${ }^{69}$, microstructural profile covariance ${ }^{47}$, and gray matter volumes ${ }^{22}$. The isocortical A-P topography resembles a frontal-polar differentiation of myelin density ${ }^{70,71}$ that situates brain regions in coherence with their functional hierarchy, i.e. a transmodal to unimodal axis ${ }^{71,72}$. Moreover, previous work has reported the topographical organisation of the $\mathrm{SiC}$ to be genetically determined ${ }^{73,74}$. The concordance of genetic similarity between the A-P subfield axis and A-P isocortical axis was previously reported using transcriptomic data ${ }^{37}$. Here, we observed even more detailed transitions of genetic similarity between individual hippocampal subregions and cortex. For instance, the posterior SUB shared genetic similarity with the unimodal isocortical regions, whereas the posterior DG did so with the transmodal isocortical regions. Differences in subfield-isocortical genetic associations may indeed relate to the subfield specific neurodevelopmental trajectories, i.e. co-development of hippocampal subfields and isocortex. For example, the CA - Ammon's Horn - is one of the first brain regions to develop in the prenatal 
period ${ }^{38,75}$ - revealing a more ambiguous genetic correlation pattern with the isocortex. Conversely, the SUB extends its maturation towards the postnatal period ${ }^{76}$, dissociating anterior transmodal and unimodal regions. Finally, DG maturation exceeds the postnatal period ${ }^{77}$, possibly underscoring posterior parietal associations. Thus, timing of pre- and post-natal development may be reflected in the genetic similarity patterning between subfields and isocortical functional domains.

Lastly, to better understand whether hippocampal function and structure underlie a common topographical pattern, in line with the structural model ${ }^{43}$, we investigated multi-modal fused gradients $G 1_{f u s}$ and $G 2_{f u s}$, and assessed local coupling of structural and functional subfield-to-isocortical maps. Whereas $G 1_{\text {fus }}$ emphasized the medial or medial/lateral portions of subfields, G2 $2_{\text {fus }}$ depicted an A-P axis in all subfields similar to the primary gradients of FC and $\mathrm{SiC}$. Indeed, $G 1_{f u s}$ may reflect a medial/lateral separation across the whole hippocampus, rather than the differentiation of individual subfields, which might emerge from the transverse axis formation as a result of cortical infolding. Possibly in line with this we observed a positive association of the primary fused gradient with T1w/T2w values, with higher T1w/T2w values in more medial portions of the subfields. This may indicate the varying microstructure in each of the subfields. Evaluating differences between structural and functional organisation, we found that coupling between structure and function was highest in posterior portions of the hippocampal subfields, whereas anterior portions were uncoupled. Indeed, we observed a consistent association between the A-P axis and structure-function coupling. Anterior, uncoupled, portions of the subfields presented on the $G 1_{F C}$ and $G 2_{f u s}$ axes were observed to be functionally connected to sensorimotor, DMN, and limbic cortices for the SUB. However, these connections became less prominent towards CA and DG. For $G 1_{S i C}$ we found anterior portions to show covariance with anterior/DMN and limbic networks, with more prominent associations with the complete isocortex towards CA and DG. In contrast, posterior portions of the subfields were more strongly functionally connected to fronto-parietal, attention, salience, and visual domains, which became more prominent towards DG, and showed structural associations with posterior temporal, attention, sensory and visual networks. Thus, mirroring observations in the cortex ${ }^{44,46}$, it may be that portions of the hippocampus associated with posterior/unimodal 
regions show more similarity between structure and function than those related to transmodal areas such as anterior frontal and temporal cortex. Functionally, the anterior hippocampus has been reported to participate in associative memory processing ${ }^{78}$, in which DMN is also involved and known to be integrating with parietal and temporal lobes for episodic memory retrieval ${ }^{79}$. Conversely, the posterior hippocampus is suggested to be a mediator for spatial memory encoding ${ }^{80}$, in which parietal cortices ${ }^{81}$ and attention and salience networks are recruited ${ }^{82}$. Together, the divergence observed in function and structure along posterior-to-anterior portions of hippocampal subfields may reflect a hierarchy of complexity, with more uncoupled portions of the hippocampus enabling more flexible forms for cognitive processing, an important hypothesis for future studies to examine.

In sum, we showed that hippocampal subfields are consistently organised along heritable anterior-to-posterior and medial-to-lateral axes which show a genetic link to isocortical functional and structural organisation. Future work may evaluate the association between maturational axes in cortical structure and divergent functional profiles along the hippocampal formation. This may provide an important step to better understand how the anatomy of the hippocampus supports its unique and versatile function. 


\section{Materials and Methods}

Participants from the HCP S900 release ${ }^{50}$ with four complete resting-state fMRI sessions and high-resolution structural images were selected. Details of subject inclusions, neuroimaging data acquisition and processing, and analysis methodology can be found in SI Appendix, Supplemental Materials and Methods. Participant recruitment procedures and informed consent forms, including consent to share de-identified data, were previously approved by the Washington University Institutional Review Board as part of the HCP. All analysis scripts used in this study are openly available at https://github.com/CNG-LAB/cngopen/tree/main/hippocampus .

We used the SurfPatch algorithm ${ }^{51}$ to automatically delineate the hippocampal subfields of all participants: subiculum (SUB), CA1-3 (CA), and CA4-DG (DG). Subfield surfaces included 1024 vertices for SUB, 2048 vertices for CA, and 1024 vertices for DG. We mapped medial sheet meshes and volumetric rs-fMRI data to native T1w space. Time series were sampled at each hippocampal and cortical mid-thickness vertex. Cortical time series were averaged within a previously established multi-modal parcellation scheme of the Glasser Atlas of 360 areas (180 regions per hemisphere) ${ }^{83}$.

For every participant separately $(n=709)$, we computed the linear correlation coefficients between isocortex-wide time series $360 \times 1200$ and hippocampal subfield time series for SUB $(1024 \times 1200)$, CA $(2048 \times 1200)$, and DG $(1024 \times 1200)$ to identify the functional connectivity (FC) matrices. The FC map of the isocortex to each hippocampal subfield for the 709 participants was mapped using linear and mixed effects models in BrainStat https://github.com/MICA-MNI/BrainStat (Fig. S2A).

Microstructural features of the hippocampus were assessed by using the ratio of T1- over T2-weighted (T1w/T2w) image intensities. Native T1w/T2w images were resampled to the MNI152 space and mapped to hippocampal subfield surfaces (SUB, CA, DG) using Connectome Workbench (v1.4.2, volume-warpfield-resample and volume-to-surface-mapping tools) ${ }^{84}$. We computed the structural intensity covariance $(\mathrm{SiC})$ by correlating hippocampal and isocortical 
T1w/T2w intensity maps resulting in a $1384 \times 1384$ matrix for SUB, $2408 \times 2408$ matrix for CA, and $1384 \times 1384$ matrix for DG.

To map the functional and microstructural organisation of hippocampal subfields, we implemented the diffusion map embedding ${ }^{52,57}$ technique on $\mathrm{FC}$ and $\mathrm{SiC}$ matrices. This approach resulted in connectome gradients, each representing a low-dimensional component of FC and $\mathrm{SiC}$ measures, separately. Gradients of $\mathrm{FC}$ and $\mathrm{SiC}$ revealed the anterior-posterior and lateral-medial axes of subfield local transitions for both modalities. We also concatenated the FC and $\mathrm{SiC}$ measures and obtained their fused gradients to identify whether different modalities support a common topographical motif together ${ }^{54}$.

Heritability and genetic correlation analysis were conducted with the Sequential Oligogenic Linkage Analysis Routines (SOLAR, v8.5.1, http://www.solar-eclipse-genetics.org). SOLAR employs a maximum likelihood variance-decomposition approach optimized to perform genetic analyses in pedigrees of arbitrary size and complexity ${ }^{55,85}$.

\section{Acknowledgements}

We would like to thank the various contributors to the open access databases that our data was downloaded from. Funding: HCP data were provided by the Human Connectome Project, Washington University, the University of Minnesota, and Oxford University Consortium (Principal Investigators: David Van Essen and Kamil Ugurbil; 1U54MH091657) funded by the 16 NIH Institutes and Centers that support the NIH Blueprint for Neuroscience Research; and by the McDonnell Center for Systems Neuroscience at Washington University. This study was supported by the Deutsche Forschungsgemeinschaft (DFG, EI 816/21-1), the National Institute of Mental Health (R01-MH074457), the Helmholtz Portfolio Theme "Supercomputing and Modeling for the Human Brain" and the European Union's Horizon 2020 Research and Innovation Program under Grant Agreement No. 785907 (HBP SGA2). R.V. was supported by the Richard and Ann Sievers award. S.L.V. was supported by Max Planck Gesellschaft (Otto Hahn award). B.C.B. acknowledges support from the SickKids Foundation (NI17-039), the National Sciences and Engineering Research Council of Canada (NSERC; Discovery-1304413), CIHR (FDN154298), Azrieli Center for Autism Research (ACAR), an MNI-Cambridge collaboration grant, and the Canada Research Chairs program. Last, this work was funded in part by Helmholtz Association's Initiative and Networking Fund under the Helmholtz International 
Lab grant agreement InterLabs-0015, and the Canada First Research Excellence Fund (CFREF Competition 2, 2015-2016) awarded to the Healthy Brains, Healthy Lives initiative at McGill University, through the Helmholtz International BigBrain Analytics and Learning Laboratory (HIBALL).

\section{Author contributions}

Ş.B., and S.L.V. conceived and designed the analysis, performed the analysis, wrote the draft manuscript and revised the manuscript. R.V. and H.L.S. aided in data analysis. A.B., B.C., N.B, R.V., and B.C.B. provided hippocampal subfield segmentations. All authors helped with writing and revising the manuscript.

\section{Competing interests}

The authors declare no conflict of interest.

\section{References}

1. Battaglia, F. P., Benchenane, K., Sirota, A., Pennartz, C. M. A. \& Wiener, S. I. The hippocampus: hub of brain network communication for memory. Trends Cogn. Sci. 15, 310-318 (2011).

2. Milner, B., Squire, L. R. \& Kandel, E. R. Cognitive Neuroscience and the Study of Memory. Neuron vol. 20 445-468 (1998).

3. Squire, L. R. Memory and the hippocampus: a synthesis from findings with rats, monkeys, and humans. Psychol. Rev. 99, 195-231 (1992).

4. Burgess, N., Maguire, E. A. \& O'Keefe, J. The human hippocampus and spatial and episodic memory. Neuron 35, 625-641 (2002).

5. Phelps, E. A. Human emotion and memory: interactions of the amygdala and hippocampal complex. Curr. Opin. Neurobiol. 14, 198-202 (2004).

6. Franklin, T. B., Saab, B. J. \& Mansuy, I. M. Neural mechanisms of stress resilience and vulnerability. Neuron 75, 747-761 (2012).

7. Pruessner, J. C. et al. Stress regulation in the central nervous system: evidence from structural and functional neuroimaging studies in human populations - 2008 Curt Richter Award Winner. Psychoneuroendocrinology 35, 179-191 (2010).

8. Lupien, S. J., McEwen, B. S., Gunnar, M. R. \& Heim, C. Effects of stress throughout the lifespan on the brain, behaviour and cognition. Nat. Rev. Neurosci. 10, 434-445 (2009).

9. Maguire, E. A. et al. Navigation-related structural change in the hippocampi of taxi drivers. Proc. Natl. Acad. Sci. U. S. A. 97, 4398-4403 (2000).

10. Palomero-Gallagher, N., Kedo, O., Mohlberg, H., Zilles, K. \& Amunts, K. Multimodal mapping and analysis of the cyto- and receptorarchitecture of the human hippocampus. Brain Struct. Funct. 225, 881-907 (2020). 
11. Wisse, L. E. M. et al. A Harmonized Segmentation Protocol for Hippocampal and Parahippocampal Subregions: Why Do We Need One and what are the Key Goals? (2017).

12. Yushkevich, P. A. et al. Quantitative comparison of 21 protocols for labeling hippocampal subfields and parahippocampal subregions in in vivo MRI: towards a harmonized segmentation protocol. Neuroimage 111, 526-541 (2015).

13. van Strien, N. M., Cappaert, N. L. M. \& Witter, M. P. The anatomy of memory: an interactive overview of the parahippocampal-hippocampal network. Nat. Rev. Neurosci. 10, 272-282 (2009).

14. de Flores, R. et al. Intrinsic connectivity of hippocampal subfields in normal elderly and mild cognitive impairment patients. Hum. Brain Mapp. 38, 4922-4932 (2017).

15. Hodgetts, C. J. et al. Ultra-High-Field fMRI Reveals a Role for the Subiculum in Scene Perceptual Discrimination. The Journal of Neuroscience vol. 37 3150-3159 (2017).

16. Berron, D. et al. Strong Evidence for Pattern Separation in Human Dentate Gyrus. $J$. Neurosci. 36, 7569-7579 (2016).

17. Neunuebel, J. P. \& Knierim, J. J. CA3 retrieves coherent representations from degraded input: direct evidence for CA3 pattern completion and dentate gyrus pattern separation. Neuron 81, 416-427 (2014).

18. Plachti, A. et al. Multimodal Parcellations and Extensive Behavioral Profiling Tackling the Hippocampus Gradient. Cereb. Cortex 29, 4595-4612 (2019).

19. Strange, B. A., Witter, M. P., Lein, E. S. \& Moser, E. I. Functional organization of the hippocampal longitudinal axis. Nat. Rev. Neurosci. 15, 655-669 (2014).

20. Bienkowski, M. S. et al. Integration of gene expression and brain-wide connectivity reveals the multiscale organization of mouse hippocampal networks. Nat. Neurosci. 21, 1628-1643 (2018).

21. Cenquizca, L. A. \& Swanson, L. W. Spatial organization of direct hippocampal field CA1 axonal projections to the rest of the cerebral cortex. Brain Res. Rev. 56, 1-26 (2007).

22. Kharabian Masouleh, S., Plachti, A., Hoffstaedter, F., Eickhoff, S. \& Genon, S. Characterizing the gradients of structural covariance in the human hippocampus. Neuroimage 218, 116972 (2020).

23. Nordin, K. et al. Structural whole-brain covariance of the anterior and posterior hippocampus: Associations with age and memory. Hippocampus 28, 151-163 (2018).

24. Maass, A., Berron, D., Libby, L. A., Ranganath, C. \& Düzel, E. Functional subregions of the human entorhinal cortex. Elife 4, (2015).

25. Genon, S., Bernhardt, B. C., La Joie, R., Amunts, K. \& Eickhoff, S. B. The many dimensions of human hippocampal organization and (dys)function. Trends Neurosci. 44, 977-989 (2021).

26. Brunec, I. K. et al. Multiple Scales of Representation along the Hippocampal Anteroposterior Axis in Humans. Curr. Biol. 28, 2129-2135.e6 (2018).

27. Chase, H. W. et al. Evidence for an anterior-posterior differentiation in the human hippocampal formation revealed by meta-analytic parcellation of fMRI coordinate maps: focus on the subiculum. Neuroimage 113, 44-60 (2015).

28. Poppenk, J., Evensmoen, H. R., Moscovitch, M. \& Nadel, L. Long-axis specialization of the human hippocampus. Trends Cogn. Sci. 17, 230-240 (2013).

29. Paquola, C. et al. Convergence of cortical types and functional motifs in the human mesiotemporal lobe. Elife 9, (2020).

30. Henriksen, E. J. et al. Spatial representation along the proximodistal axis of CA1. Neuron 
68, 127-137 (2010).

31. Li, Q. et al. Human brain function during pattern separation follows hippocampal and neocortical connectivity gradients. doi:10.1101/2020.06.22.165290.

32. Przeździk, I., Faber, M., Fernández, G., Beckmann, C. F. \& Haak, K. V. The functional organisation of the hippocampus along its long axis is gradual and predicts recollection. Cortex 119, 324-335 (2019).

33. Vos de Wael, R. et al. Anatomical and microstructural determinants of hippocampal subfield functional connectome embedding. Proc. Natl. Acad. Sci. U. S. A. 115, 10154-10159 (2018).

34. Haak, K. V. \& Beckmann, C. F. Understanding brain organisation in the face of functional heterogeneity and functional multiplicity. Neuroimage 220, 117061 (2020).

35. Huntenburg, J. M., Bazin, P.-L. \& Margulies, D. S. Large-Scale Gradients in Human Cortical Organization. Trends Cogn. Sci. 22, 21-31 (2018).

36. Margulies, D. S. et al. Situating the default-mode network along a principal gradient of macroscale cortical organization. Proc. Natl. Acad. Sci. U. S. A. 113, 12574-12579 (2016).

37. Vogel, J. W. et al. A molecular gradient along the longitudinal axis of the human hippocampus informs large-scale behavioral systems. Nature Communications vol. 11 (2020).

38. Whelan, C. D. et al. Heritability and reliability of automatically segmented human hippocampal formation subregions. Neuroimage 128, 125-137 (2016).

39. Patel, S. et al. Heritability of hippocampal subfield volumes using a twin and non-twin siblings design. Hum. Brain Mapp. 38, 4337-4352 (2017).

40. van der Meer, D. et al. Brain scans from 21,297 individuals reveal the genetic architecture of hippocampal subfield volumes. Mol. Psychiatry 25, 3053-3065 (2020).

41. Bannerman, D. M. et al. Hippocampal synaptic plasticity, spatial memory and anxiety. Nat. Rev. Neurosci. 15, 181-192 (2014).

42. McEwen, B. S. STRESS AND HIPPOCAMPAL PLASTICITY. Annual Review of Neuroscience vol. 22 105-122 (1999).

43. Barbas, H. General cortical and special prefrontal connections: principles from structure to function. Annu. Rev. Neurosci. 38, 269-289 (2015).

44. Valk, S. L. et al. Genetic and phylogenetic uncoupling of structure and function in human transmodal cortex. doi:10.1101/2021.06.08.447522.

45. Suárez, L. E., Markello, R. D., Betzel, R. F. \& Misic, B. Linking Structure and Function in Macroscale Brain Networks. Trends Cogn. Sci. 24, 302-315 (2020).

46. Baum, G. L. et al. Development of structure-function coupling in human brain networks during youth. Proc. Natl. Acad. Sci. U. S. A. 117, 771-778 (2020).

47. Paquola, C. et al. Microstructural and functional gradients are increasingly dissociated in transmodal cortices. PLoS Biol. 17, e3000284 (2019).

48. Buckner, R. L. \& Krienen, F. M. The evolution of distributed association networks in the human brain. Trends Cogn. Sci. 17, 648-665 (2013).

49. Mesulam, M.-M. Principles of Behavioral and Cognitive Neurology. (Oxford University Press, 2000).

50. Van Essen, D. C. et al. The WU-Minn Human Connectome Project: an overview. Neuroimage 80, 62-79 (2013).

51. Caldairou, B. et al. A Surface Patch-Based Segmentation Method for Hippocampal Subfields. Medical Image Computing and Computer-Assisted Intervention-MICCAI 2016 
379-387 (2016) doi:10.1007/978-3-319-46723-8 44.

52. Coifman, R. R. \& Lafon, S. Diffusion maps. Applied and Computational Harmonic Analysis vol. 21 5-30 (2006).

53. Glasser, M. F. \& Van Essen, D. C. Mapping human cortical areas in vivo based on myelin content as revealed by T1- and T2-weighted MRI. J. Neurosci. 31, 11597-11616 (2011).

54. Paquola, C. et al. A multi-scale cortical wiring space links cellular architecture and functional dynamics in the human brain. PLOS Biology vol. 18 e3000979 (2020).

55. Almasy, L. \& Blangero, J. Multipoint quantitative-trait linkage analysis in general pedigrees. Am. J. Hum. Genet. 62, 1198-1211 (1998).

56. Burt, J. B., Helmer, M., Shinn, M., Anticevic, A. \& Murray, J. D. Generative modeling of brain maps with spatial autocorrelation. Neuroimage 220, 117038 (2020).

57. Vos de Wael, R. et al. BrainSpace: a toolbox for the analysis of macroscale gradients in neuroimaging and connectomics datasets. Commun Biol 3, 103 (2020).

58. DeKraker, J., Köhler, S. \& Khan, A. R. Surface-based hippocampal subfield segmentation. Trends Neurosci. 44, 856-863 (2021).

59. Bernhardt, B. C. et al. The spectrum of structural and functional imaging abnormalities in temporal lobe epilepsy. Ann. Neurol. 80, 142-153 (2016).

60. Elman, J. A. et al. Genetic architecture of hippocampal subfields on standard resolution MRI: How the parts relate to the whole. Hum. Brain Mapp. 40, 1528-1540 (2019).

61. Hibar, D. P. et al. Novel genetic loci associated with hippocampal volume. Nat. Commun. 8, 13624 (2017).

62. Hibar, D. P. et al. Common genetic variants influence human subcortical brain structures. Nature 520, 224-229 (2015).

63. Stein, J. L. et al. Identification of common variants associated with human hippocampal and intracranial volumes. Nat. Genet. 44, 552-561 (2012).

64. Maller, J. J. et al. Hippocampal volumetrics in treatment-resistant depression and schizophrenia: the devil's in de-tail. Hippocampus 22, 9-16 (2012).

65. Arnatkeviciute, A. et al. Genetic influences on hub connectivity of the human connectome. Nat. Commun. 12, 4237 (2021).

66. Haak, K. V. \& Beckmann, C. F. Plasticity versus stability across the human cortical visual connectome. Nat. Commun. 10, 3174 (2019).

67. Cooper, C. 'iana, Moon, H. Y. \& van Praag, H. On the Run for Hippocampal Plasticity. Cold Spring Harb. Perspect. Med. 8, (2018).

68. Alexander-Bloch, A., Raznahan, A., Bullmore, E. \& Giedd, J. The convergence of maturational change and structural covariance in human cortical networks. J. Neurosci. 33, 2889-2899 (2013).

69. Valk, S. L. et al. Shaping brain structure: Genetic and phylogenetic axes of macroscale organization of cortical thickness. Sci Adv 6, (2020).

70. Burt, J. B. et al. Hierarchy of transcriptomic specialization across human cortex captured by structural neuroimaging topography. Nature Neuroscience vol. 21 1251-1259 (2018).

71. Cahalane, D. J., Charvet, C. J. \& Finlay, B. L. Modeling local and cross-species neuron number variations in the cerebral cortex as arising from a common mechanism. Proc. Natl. Acad. Sci. U. S. A. 111, 17642-17647 (2014).

72. Fornito, A., Arnatkevičiūtè, A. \& Fulcher, B. D. Bridging the Gap between Connectome and Transcriptome. Trends Cogn. Sci. 23, 34-50 (2019).

73. Alexander-Bloch, A. F. et al. Human Cortical Thickness Organized into 
Genetically-determined Communities across Spatial Resolutions. Cereb. Cortex 29, 106-118 (2019).

74. Chen, C.-H. et al. Genetic topography of brain morphology. Proc. Natl. Acad. Sci. U. S. A. 110, 17089-17094 (2013).

75. Taupin, P. The Hippocampus: Neurotransmission and Plasticity in the Nervous System. (Nova Publishers, 2007).

76. Jabès, A., Lavenex, P. B., Amaral, D. G. \& Lavenex, P. Postnatal development of the hippocampal formation: a stereological study in macaque monkeys. J. Comp. Neurol. 519, 1051-1070 (2011).

77. Insausti, R., Cebada-Sánchez, S. \& Marcos, P. Postnatal Development of the Human Hippocampal Formation. Advances in Anatomy, Embryology and Cell Biology (2010) doi:10.1007/978-3-642-03661-3.

78. Buzsáki, G. \& Tingley, D. Space and Time: The Hippocampus as a Sequence Generator. Trends Cogn. Sci. 22, 853-869 (2018).

79. Wagner, A. D., Shannon, B. J., Kahn, I. \& Buckner, R. L. Parietal lobe contributions to episodic memory retrieval. Trends Cogn. Sci. 9, 445-453 (2005).

80. Fanselow, M. S. \& Dong, H.-W. Are the dorsal and ventral hippocampus functionally distinct structures? Neuron 65, 7-19 (2010).

81. Save, E., Poucet, B., Foreman, N. \& Buhot, M.-C. Object exploration and reactions to spatial and nonspatial changes in hooded rats following damage to parietal cortex or hippocampal formation. Behavioral Neuroscience vol. 106 447-456 (1992).

82. Corbetta, M. \& Shulman, G. L. Spatial neglect and attention networks. Annu. Rev. Neurosci. 34, 569-599 (2011).

83. Glasser, M. F. et al. A multi-modal parcellation of human cerebral cortex. Nature 536, 171-178 (2016).

84. Marcus, D. S. et al. Informatics and data mining tools and strategies for the human connectome project. Front. Neuroinform. 5, 4 (2011).

85. Kochunov, P. et al. Homogenizing Estimates of Heritability Among SOLAR-Eclipse, OpenMx, APACE, and FPHI Software Packages in Neuroimaging Data. Front. Neuroinform. 13, 16 (2019). 


\section{Supplementary Information}

\section{Participants}

Participants with anatomical anomalies or tissue segmentation errors listed in the HCP issues were discarded $(\mathrm{n}=40)$. For every participant, we segmented hippocampal subfields: subiculum (SUB), CA1-3 (CA), and CA4-DG (DG) along the structural images using a patch-based surface algorithm ${ }^{44}$. Based on a visual inspection of subfield delineations, we discarded participants with poor segmentation quality $(n=42)$. As a necessity for the functional connectivity $(\mathrm{FC})$ gradient analysis (see section Functional Connectivity and Gradients), we further excluded participants $(\mathrm{n}=31)$, whose FC maps were poorly associated with the group-level reference FC. There remained $n=709$ participants (395 women, mean \pm SD age $=28.7 \pm 3.7 \mathrm{y}$ ) accessible for our study. Among the 709 participants included in this study, there were 176 monozygotic twins, 178 siblings without twin status and 355 participants without familial relatedness. All quality assessment steps and analysis scripts used in this study are available at https://github.com/CNG-LAB/cngopen/tree/main/hippocampus.

\section{Neuroimaging Data Acquisition and Preprocessing}

Details of the HCP neuroimaging protocol and processing pipelines are available at ${ }^{81}$ ). In brief, we extracted T1-weighted (T1w) and T2-weighted (T2w) images available in the HCP initiative, which were all acquired on a 3T Siemens Skyra scanner. T1w images were acquired using a three-dimensional magnetization prepared rapid gradient-echo (3D-MPRAGE) sequence $(0.7 \mathrm{~mm}$ isotropic voxels, matrix $=320 \times 320,256$ sagittal slices, $\mathrm{TR}=2400 \mathrm{~ms}, \mathrm{TE}=2.14 \mathrm{~ms}$, $\mathrm{TI}=1000 \mathrm{~ms}$, flip angle $=8^{\circ}$, iPAT $=2$ ). Resting-state fMRI images were acquired using a multi-band accelerated 2D-BOLD echo-planar imaging (EPI) sequence ( $2 \mathrm{~mm}$ isotropic voxels, matrix $=104 \times 90,72$ sagittal slices, $\mathrm{TR}=720 \mathrm{~ms}, \mathrm{TE}=33 \mathrm{~ms}$, flip angle $=52^{\circ}, \mathrm{mb}$ factor $=8$, 1200 volumes/scan). The fMRI data was collected at two sessions $(1,2)$ and in two phase encoding directions at each session (left-right [LR] and right-left [RL]), resulting in four resting-state fMRI datasets in total ([LR1], [RL1], [LR2], [RL2]). 
Preprocessing steps for the structural MRI images included gradient nonlinearity correction, brain extraction, distortion correction and co-registration of $\mathrm{T} 1 \mathrm{w}$ and $\mathrm{T} 2 \mathrm{w}$ images using rigid body transformations. Then, an intensity nonuniformity correction was performed using T1w and T2w contrasts ${ }^{41}$ and subcortical structures were segmented using FSL FIRST ${ }^{82}$. Subsequently, preprocessed images were nonlinearly registered to the MNI152 template and cortical surfaces were reconstructed with FreeSurfer 5.3.0-HCP ${ }^{83-85}$. Finally, the individual cortical surfaces were registered to the Conte69 template ${ }^{86}$ using MSMA11 ${ }^{87}$.

Preprocessing of rs-fMRI images included corrections for the gradient nonlinearity, head motion and distortion. The images were then aligned to the $\mathrm{T} 1 \mathrm{w}$ space using rigid-body and boundary-based registrations together ${ }^{88}$. The transformation matrices from this alignment step and that of the earlier T2w to T1w alignment were concatenated and applied to the rs-fMRI images at a single interpolation step to warp rs-fMRI images to the MNI152. Further processing in MNI152 space included bias field removal, whole brain intensity normalization, high pass filtering ( $>2000$ s FWHM) and noise removal with the ICA-FIX procedure ${ }^{89}$.

\section{Hippocampus Subfield Segmentations}

We used the SurfPatch algorithm ${ }^{44}$ to automatically delineate the hippocampal subfields of all participants: subiculum (SUB), CA1-3 (CA), and CA4-DG (DG). SurfPatch is a multi-template surface-path hippocampal segmentation method trained on a public dataset of manual segmentations in healthy controls ${ }^{90}$, and has been validated in patients with histopathology of the hippocampus ${ }^{52}$. This algorithm incorporates a spherical harmonic shape parameterization and point distribution model of the surface ${ }^{91}$. Next, to minimize partial volume effects, we generated medial surfaces running through the center of each subfield using a Hamilton-Jakobian approach ${ }^{92}$. The spherical harmonic parameterization was propagated to the medial sheet to improve vertex-correspondence across individuals based on shape inherent information. Resultant CA surfaces consisted of 10242 vertices and both DG and SUB surfaces of 5762 vertices. Next, CA, DG, and SUB surfaces were further downsampled to 2048, 1024, and 1024 vertices, respectively. All surface segmentations underwent a visual inspection and are available upon request. 


\section{Isocortex and Subfield Time Series}

We mapped medial sheet meshes and volumetric resting-state fMRI data to native $\mathrm{T} 1 \mathrm{w}$ space. Time series were sampled at each hippocampal and cortical mid-thickness vertex ${ }^{87}$. Hippocampal surface features were smoothed using a Gaussian diffusion kernel with 5 mesh units as FWHM in all subfields and isocortices. Sampling was carried out in a native T1w space to minimize the interpolation. Cortical time series were averaged within a previously established multi-modal parcellation scheme of the Glasser Atlas of 360 areas (180 regions per hemisphere) ${ }^{87}$. Surface-based time series were smoothed using a Gaussian diffusion kernel with 5 mesh units as full-width-at-half-maximum (FWHM).

\section{Functional Connectivity}

For every participant separately $(\mathrm{n}=740)$, we computed the linear correlation coefficients between isocortex-wide time series $(360 \times 1200)$ and hippocampal subfield time series for SUB $(1024 \times 1200)$, CA $(2048 \times 1200)$, and DG $(1024 \times 1200)$. This resulted in a isocortex wide functional connectivity $(\mathrm{FC})$ map $(360 \times 1)$ for every subject and subfield. We obtained group-level reference FC maps for every subfield by averaging individual FC maps across participants. We further profiled the similarity of individual FC maps to the reference FC maps by means of simple correlation (Supplementary Fig. S1A). Participants with a lower degree of similarity $(r<0.45)$ to the reference map were excluded $(n=31)$. Finally, the FC map of the isocortex to each hippocampal subfield for the remaining 709 participants was mapped using linear and mixed effects models in BrainStat (https://github.com/MICA-MNI/BrainStat).

\section{T1w/T2w Maps and Structural Intensity Covariance}

To study microstructural features of the hippocampus, we used the ratio of T1- over T2-weighted (T1w/T2w) image intensities. We resampled native $\mathrm{T} 1 \mathrm{w} / \mathrm{T} 2 \mathrm{w}$ images to the MNI152 space and mapped them to hippocampal subfield surfaces (SUB, CA, DG) using Connectome Workbench (v1.4.2, volume-warpfield-resample and volume-to-surface-mapping 
tools) ${ }^{93}$. To assess the quality of $\mathrm{T} 1 \mathrm{w} / \mathrm{T} 2 \mathrm{w}$ intensities projected on the hippocampal subfields, we obtained the mean $\mathrm{T} 1 \mathrm{w} / \mathrm{T} 2 \mathrm{w}$ intensity distributions of all participants for potential outlier detection (Fig. 2A, Supplementary Fig. S3C). We computed the structural intensity covariance (SiC) by correlating hippocampal and cortical T1w/T2w intensity maps resulting in $1384 \times 1384$ matrix for SUB, $2408 \times 2408$ matrix for CA, and $1384 \times 1384$ matrix for DG.

\section{Heritability and Genetic Correlation}

Heritability and genetic correlation analysis were conducted with the Sequential Oligogenic Linkage Analysis Routines (SOLAR, v8.5.1, http://www.solar-eclipse-genetics.org/). SOLAR employs a maximum likelihood variance-decomposition approach optimized to perform genetic analyses in pedigrees of arbitrary size and complexity ${ }^{45,94}$. SOLAR models genetic proximity by covariance between family members ${ }^{45,94}$.

In brief, heritability (i.e. narrow-sense heritability $h^{2}$ ) is defined as the proportion of the phenotypic variance $\left(\sigma_{p}^{2}\right)$ in a trait that is attributable to the additive effects of genes $\left(\sigma_{g}^{2}\right)$, i.e. $h^{2}=\sigma_{g}^{2} / \sigma_{p}^{2}$. SOLAR estimates heritability by comparing the observed phenotypic covariance matrix with the covariance matrix predicted by kinship ${ }^{45,94}$. Significance of the heritability estimate was tested using a likelihood ratio test where the likelihood of a restricted model (with $\sigma_{g}^{2}$ constrained to zero) is compared with the likelihood of the estimated model. Twice the difference between the log likelihoods of these models yields a test statistic, which is asymptotically distributed as a 50:50 mixture of a $\chi^{2}$ variable with 1 degree-of-freedom and a point mass at zero ${ }^{45,94}$. We quantified the heritability of (i) hippocampal-isocortical functional connectivity patterns, (ii) hippocampal subfield gradients, and (iii) T1w/T2w intensity maps. We included covariates in all heritability analyses including age, sex, age $\times$ sex, age and $a g e^{2} \times \operatorname{sex}$

To estimate if variations in T1w/T2w intensity maps between hippocampus and isocortex were influenced by the same genetic factors, a genetic correlation analysis was conducted. 
Genetic correlations indicate the proportion of variance that determines the extent to which genetic influences on one trait are shared with genetic influences on another trait (e.g. pleiotropy). In SOLAR, the phenotypic correlation $\left(\rho_{p}\right)$ was decomposed through bivariate polygenic analyses to estimate genetic $\left(\rho_{g}\right)$ and environmental $\left(\rho_{e}\right)$ correlations using the following formula: $\rho_{p}=\rho_{g} \sqrt{h_{1}^{2} h_{2}^{2}}+\rho_{e} \sqrt{\left(1-h_{1}^{2}\right)\left(1-h_{2}^{2}\right)}$, where $h_{1}^{2}$ and $h_{2}^{2}$ are the heritability estimates of the vertex-based values in hippocampus and isocortex ${ }^{95,96}$. The significance of these correlations was determined (similar to heritability analyses) by likelihood ratio tests comparing a model in which $\rho_{g}$ was estimated with a model in which $\rho_{g}$ was constrained to zero (no shared genetic effect) and constrained to 1 (complete pleiotropy) ${ }^{95,96}$.

\section{Connectivity Gradients}

We computed hippocampal subfield FC gradients, similarly to those identified by Vos de Wael (2018). Here, for every participant $(n=709)$, we first concatenated the subfield times series across SUB, CA, and DG. Next, the time series of each vertex was correlated with the isocortex wide time series, yielding a hippocampal-cortical FC map $(4096 \times 360)$ for every subject. We used BrainSpace ${ }^{31,47}$ to derive connectivity gradients from the group-level FC matrix with the diffusion map embedding (normalized angle kernel, 90th percentile thresholding for the sparsity, and diffusion time estimation of $\alpha=0.5)^{40}$. This algorithm is in fact a manifold learning method that reveals underlying spatial variations in a connected graph, i.e. in our case in the connectivity patterns of the hippocampal surfaces when moving from one vertex to another.

Using the diffusion embedding algorithm, we generated low-dimensional representations of hippocampal-cortical FC, namely the gradients. Along each single gradient $(4096 \times 1)$, hippocampus vertices that share similar connectivity patterns have similar embedding values. The first and second gradients explained $32.4 \%$ of the total variance in the subfield FC map (Supplementary Fig. S1B, S1C). Having validated the gradient representations of hippocampal subfields at the group-level, we computed the individual-level gradients for every participant. Subsequently, individual gradients were aligned to the group-level gradients using Procrustes alignment to be scaled onto a common embedded connectivity space. 
The gradient approach was further implemented on the heritability scores, structural intensity covariance $(\mathrm{SiC})$, and genetic correlation measures individually, which aimed to characterize spatial motifs underlined by each of these modalities. We also implemented the fused gradients approach to identify whether different modalities support a common topographical motif together ${ }^{42}$. FC and $\mathrm{SiC}$ matrices were concatenated horizontally and rescaled to identical ranges to generate the fused data. This way we aimed to combine different modalities by still preserving the unique information in each. Secondly, performing the diffusion embedding manifold learning on the fused data, we obtained multimodal gradients driven by FC and $\mathrm{SiC}$ simultaneously.
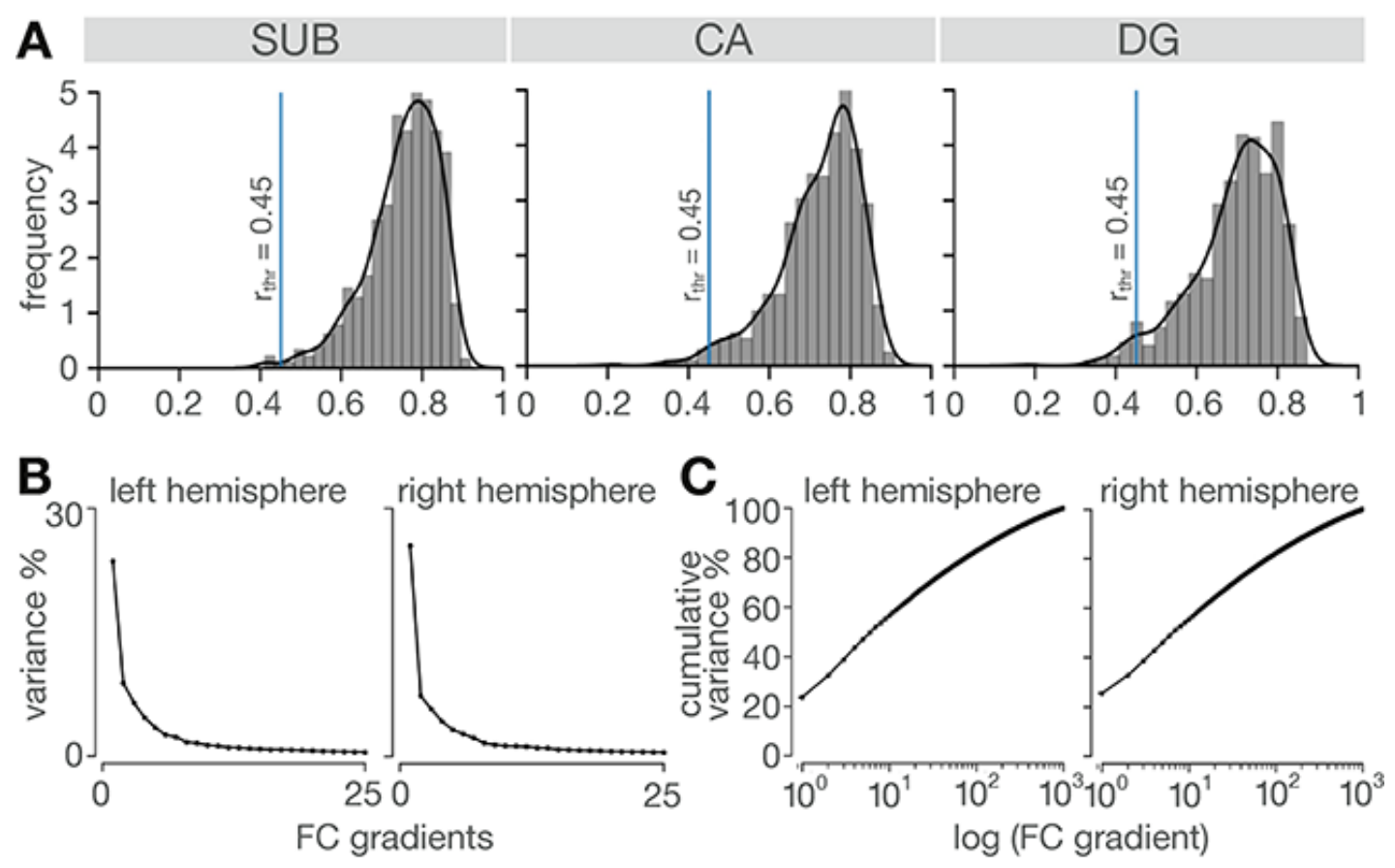

Supplementary Fig. S1. Similarity of individual hippocampal-isocortical FC maps to the group-level FC and variance explained by hippocampal FC gradients. a. FC similarity of $n=740$ participants to the group-level FC quantified by means of Pearson's correlations (r). Threshold r-value $\left(r_{t h r}=0.45\right)$ was assessed by computing the 2.5 standard deviation distance away from the mean r-values. Participants with a lower degree of similarity $\left(r_{t h r}<0.45, \mathrm{n}=31\right)$ were excluded prior to the functional connectome gradient analysis. b. The first gradients explained $23.6 \%$ of total hippocampal-isocortical FC variance for the left hemisphere (left) and $25.5 \%$ for the right hemisphere (right). c. Cumulative variance explained by the first three gradients was $38.5 \%$ for the left hemisphere (left) and 38.9\% for the right hemisphere (right). 
A Hippocampal-isocortical connectivity analysis for subfields
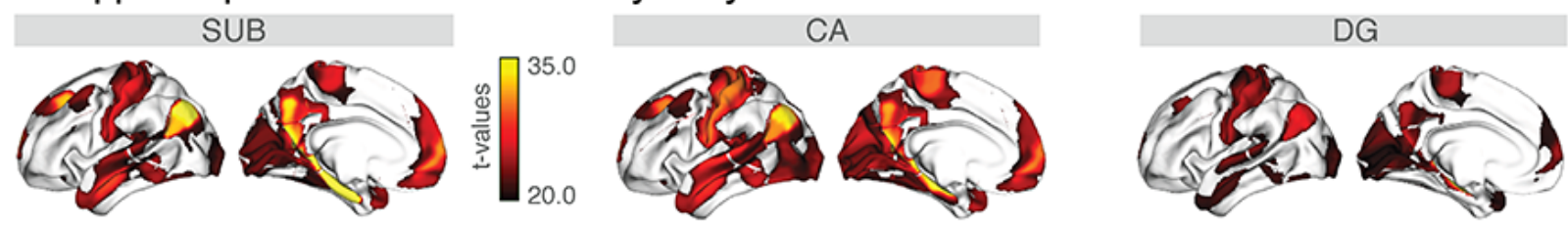

B Heritability of hippocampal-isocortical functional connectivity
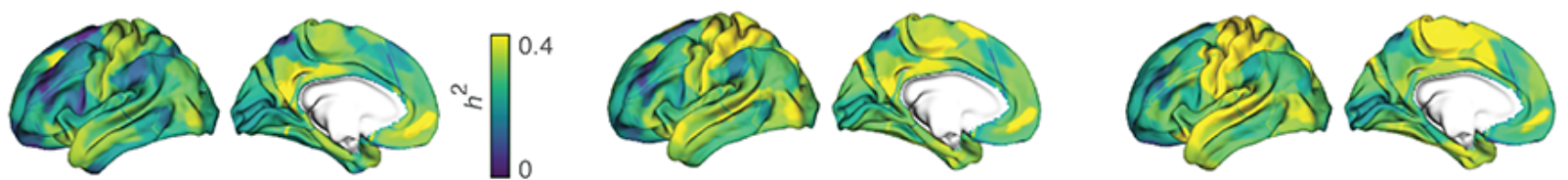

C Significance levels of the hippocampal-isocortical functional connectivity heritability
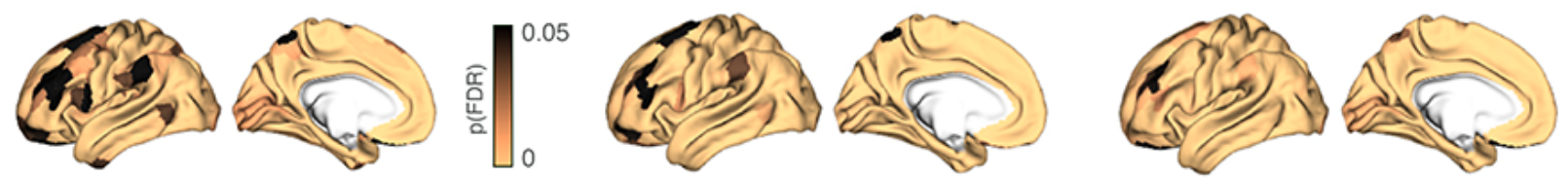

D Network based functional couplings and their heritability scores
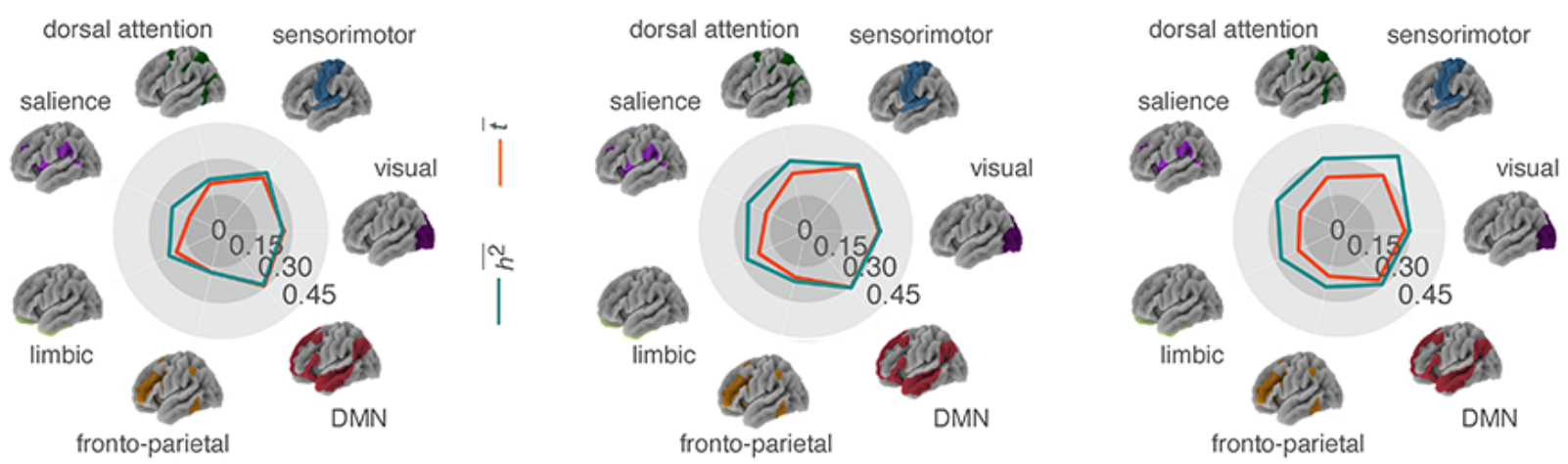

E Significance levels of the functional gradient heritability
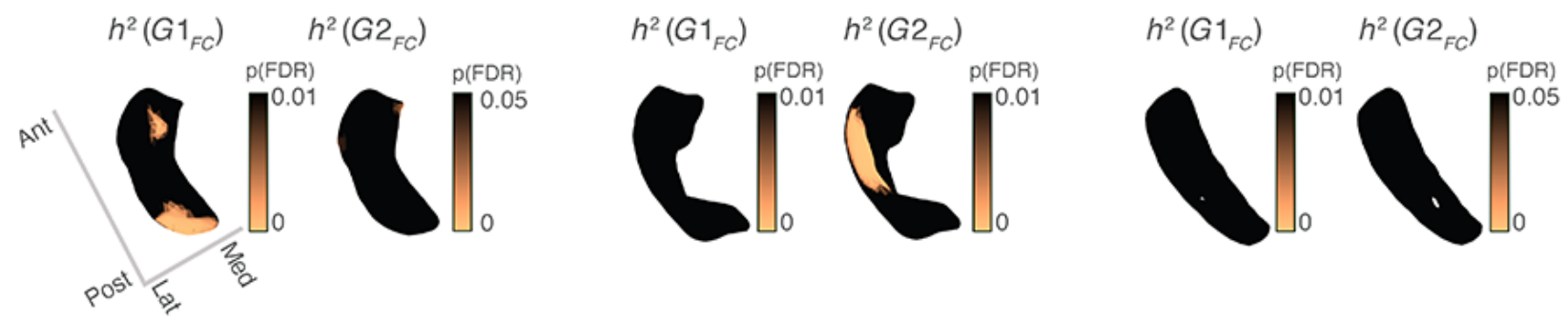

Supplementary Fig. S2. Hippocampal-isocortical functional organisation and its heritability at the individual subfield level. A. Isocortex-wide functional connectivity (FC) of SUB (left), CA (middle), and DG (right). Isocortex-wide findings were thresholded at $\mathrm{t}>20$ to represent the highest connections. B. Heritability $\left(h^{2}\right)$ scores of the hippocampal-isocortical functional couplings throughout the cortex. C. Significance levels of the $h^{2}$ scores from panel B. Significance level was reported with the multiple comparison corrected p-values $(\mathrm{p}(\mathrm{FDR}))$. Copper color denotes $\mathrm{pFDR}<0.05$ and black pFDR $\geq 0.05$. D. Isocortical-hippocampal FC strength and its heritability (t-values $\& h^{2}$ scores) distributed into seven networks ${ }^{1}$ and averaged $\left(E\right.$ and $\overline{h^{2}}$ ). E. Significance levels of gradient map heritability $\left(h^{2}\left(G 1_{F C}\right)\right.$ and $\left.h^{2}\left(G 2_{F C}\right)\right)$ reported by p(FDR). 
A

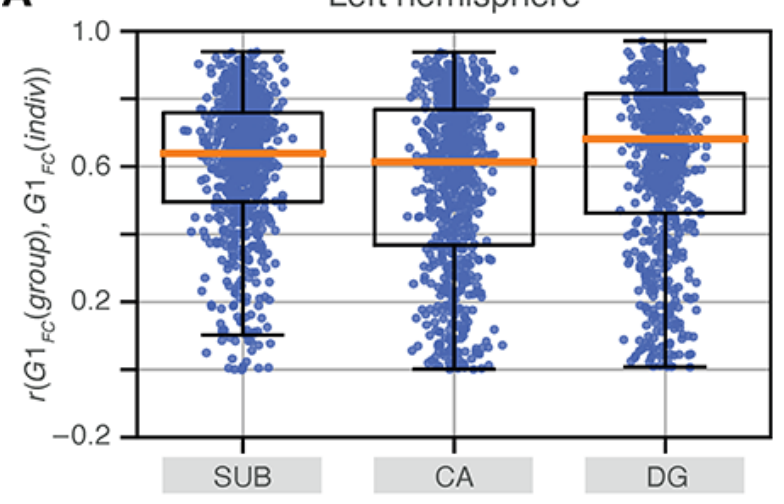

C

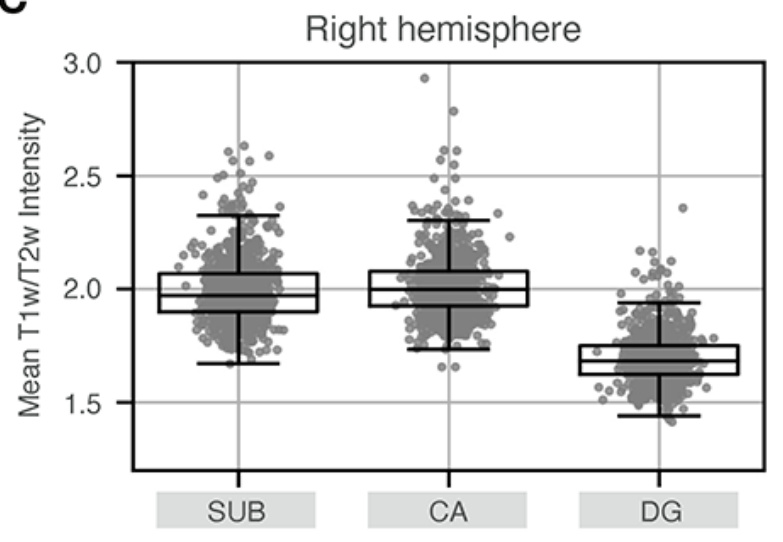

B

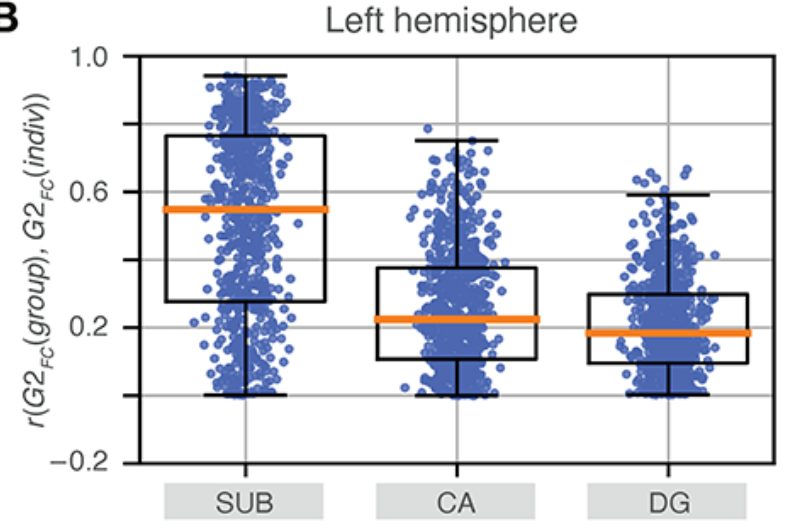

D

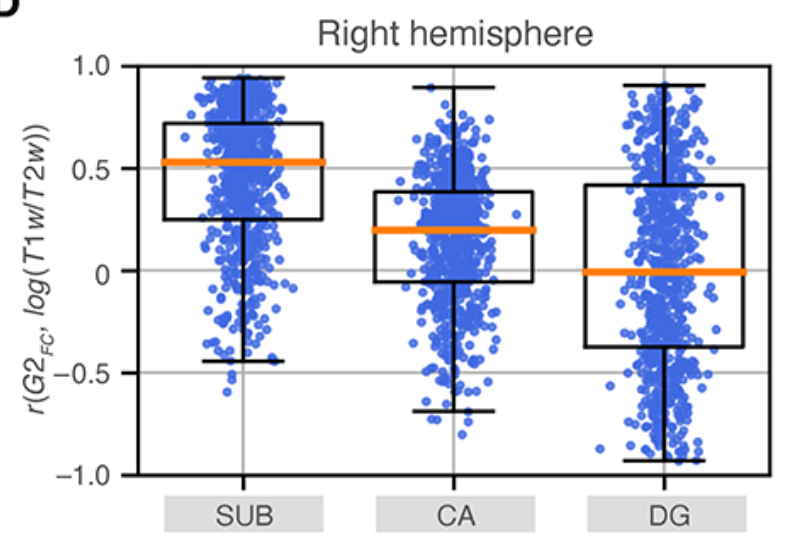

Supplementary Fig. S3. Quality assessment on primary and secondary functional gradients $\left(G 1_{F C}\right.$, $G 2_{F C}$ ) as well as T1w/T2w intensity maps. A. Pearson correlations (y-axis) between the group-level $G 1_{F C}$ map and individual-level $G 1_{F C}$ maps $(\mathrm{n}=709)$ for each subfield (SUB, CA, and DG, x-axis) in the left hemisphere. Individual correlations were significantly positive for the SUB (median $\bar{r}=0.64, \mathrm{p}<$ 0.001, one-tailed Wilcoxon signed-rank test), CA $(\bar{r}=0.61, \mathrm{p}<0.001)$, and DG $(\bar{r}=0.68, \mathrm{p}<0.001)$. B. Pearson correlations (y-axis) between the group-level $G 2_{F C}$ map and individual-level $G 2_{F C}$ maps for each subfield (x-axis) in the left hemisphere. Individual correlations were significantly positive for the SUB $(\bar{r}$ $=0.55, \mathrm{p}<0.001), \mathrm{CA}(\bar{r}=0.22, \mathrm{p}<0.001)$, and DG $(\bar{r}=0.18, \mathrm{p}<0.001)$. C. Mean T1w/T2w intensity map distributions for each subject (x-axis) and each subfield (y-axis) in the right hemisphere. Mean and standard deviations of T1w/T2w maps were $1.99 \pm 0.43$ for SUB, $2.01 \pm 0.68$ for CA, and $1.69 \pm 0.23$ for DG. D. Individual-level Pearson correlations (y-axis) between $G 2_{F C}$ maps and T1w/T2w intensity maps for each subfield (x-axis) in the right hemisphere. Individual correlations were significantly positive for the SUB $(\bar{r}=0.53, \mathrm{p}<0.001)$ and CA $(\bar{r}=0.20, \mathrm{p}<0.001)$, however, not for DG $(\bar{r}=-0.01$, $\mathrm{p}=0.6$ ). 
A
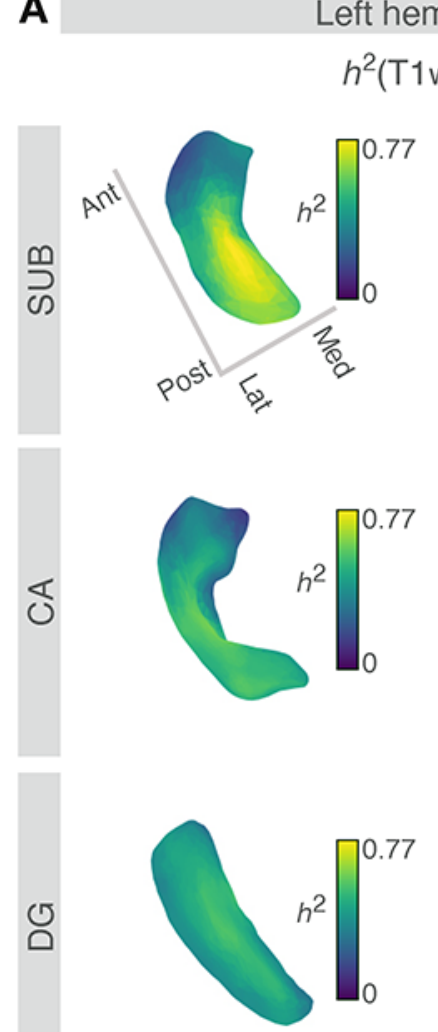
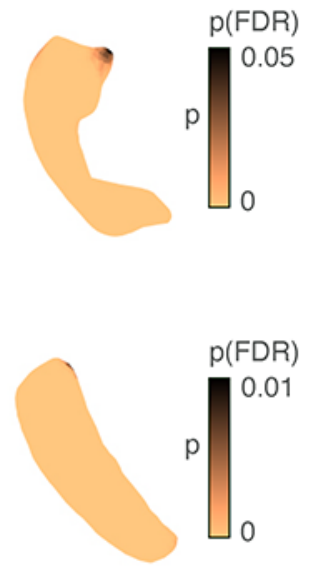

B
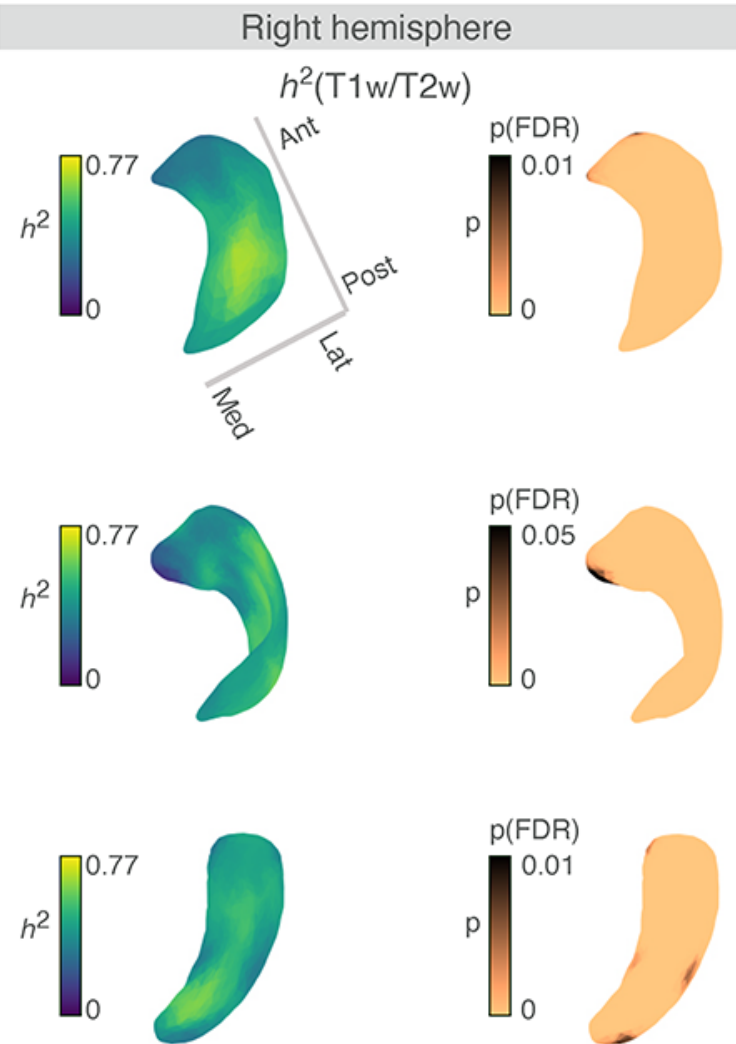

Supplementary Fig. S4. Heritability of the T1w/T2w intensity maps $\left(h^{2}(\mathrm{~T} 1 \mathrm{w} / \mathrm{T} 2 \mathrm{w})\right)$ by controlling for the mean T1w/T2w intensities for each subfield (SUB, CA, DG) and hemisphere (left and right). A. $h^{2}(\mathrm{~T} 1 \mathrm{w} / \mathrm{T} 2 \mathrm{w})$ patterns and their FDR-corrected significance levels ( $\left.\mathrm{p}(\mathrm{FDR})\right)$ did not change after controlling for the mean $\mathrm{T} 1 \mathrm{w} / \mathrm{T} 2 \mathrm{w}$ intensities for the left hemisphere (copper color denotes $\mathrm{pFDR}<0.05$, black pFDR > 0.05). B. Similar results were observed for the right hemisphere. 
A Isocortical projections of primary fused gradient

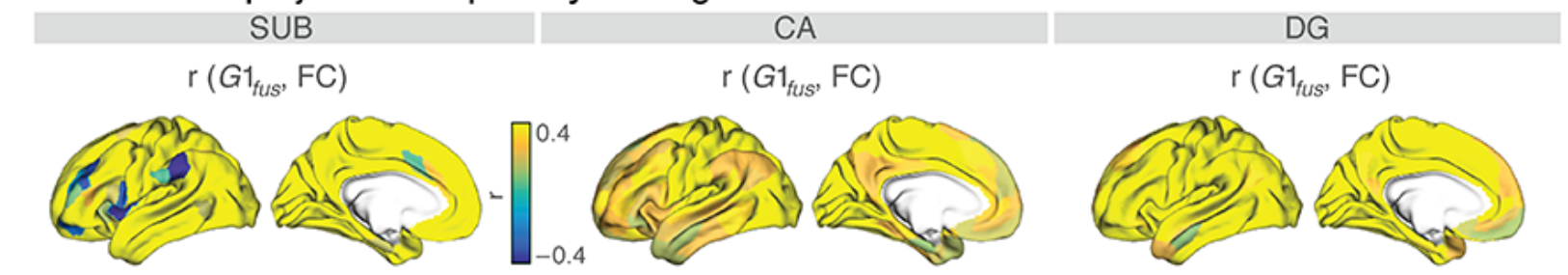

B Isocortical projections of secondary fused gradient

$r\left(G 2_{\text {fus }}, F C\right)$
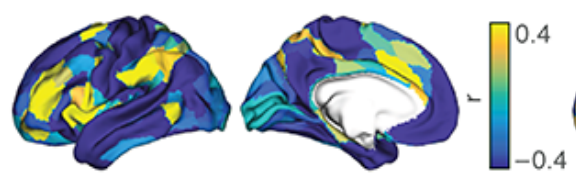

$r\left(G 2_{\text {fus }}, \mathrm{FC}\right)$

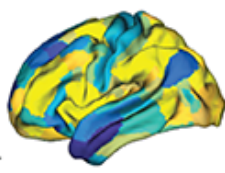

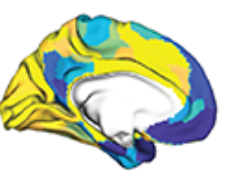

$r\left(G 2_{f u s}, F C\right)$
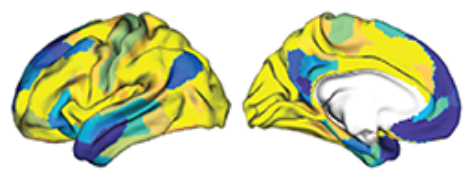

C Network-level functional couplings of anterior and posterior subfield portions described by $G 2_{\text {fus }}$
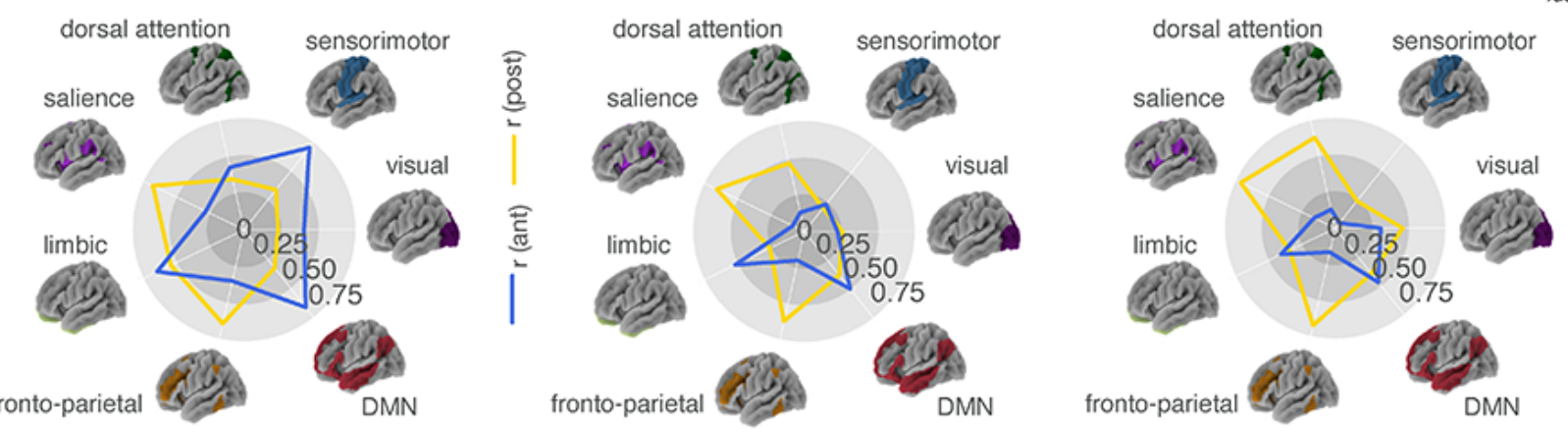

D Isocortical projections of secondary functional connectivity gradient
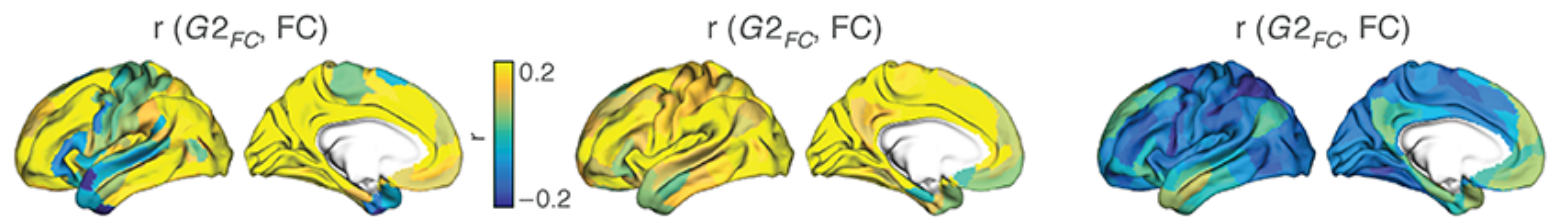

E Isocortical projections of secondary structural intensity covariance gradient
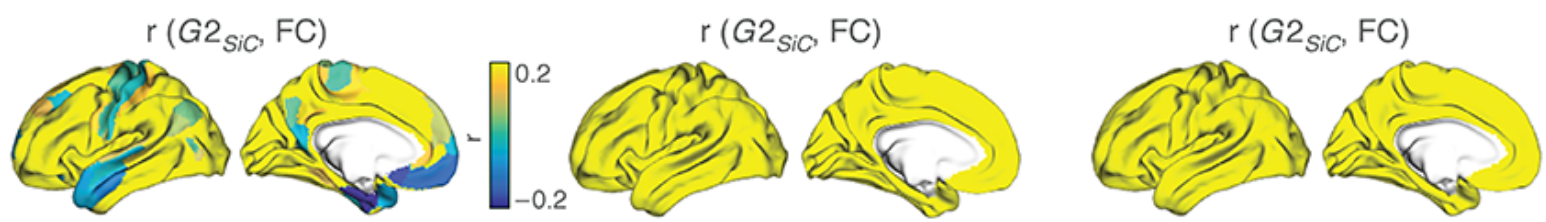

F Isocortical projections of secondary genetic correlation gradient
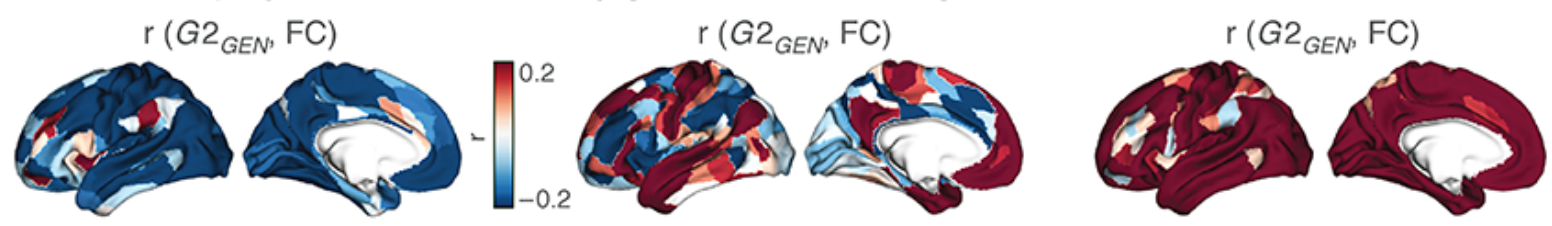

Supplementary Fig. S5. A. Variations in hippocampal-isocortical functional connectivity (FC) across the principal fused gradient $\left(G 1_{f u s}\right)$ projected onto the isocortex (r-values). B. Variations in hippocampal-isocortical FC across the secondary fused gradient $\left(G 2_{f u s}\right)$ projected onto the isocortex. $\mathbf{C}$. 
G2 fus -isocortical projections in Paned B visualized across the large-scale functional networks. $\mathrm{r}$ (ant) indicate average functional couplings between anterior subfield portions and the isocortex in each network, $r$ (post) depict that of posterior subfield portions and the isocortex. D. Variations in hippocampal-isocortical FC across the secondary FC gradient $\left(G 2_{F C}\right)$ projected onto the isocortex. E. Variations in hippocampal-isocortical FC across the secondary structural intensity covariance gradient ( $G 2_{\text {SiC }}$ ) projected onto the isocortex. F. Variations in hippocampal-isocortical FC across the secondary genetic correlations gradient $\left(G 2_{G E N}\right)$ projected onto the isocortex.

A Macroscale functional gradients of the isocortex
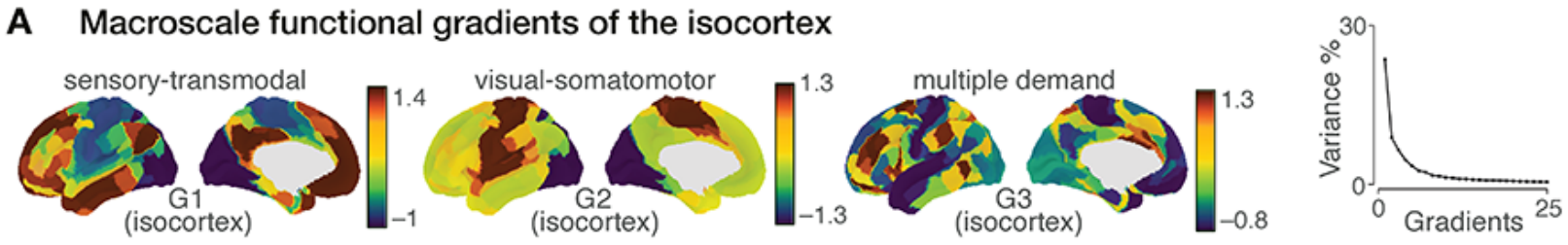

B Spin permutation testing for the large scale gradient assocations across the isocortex
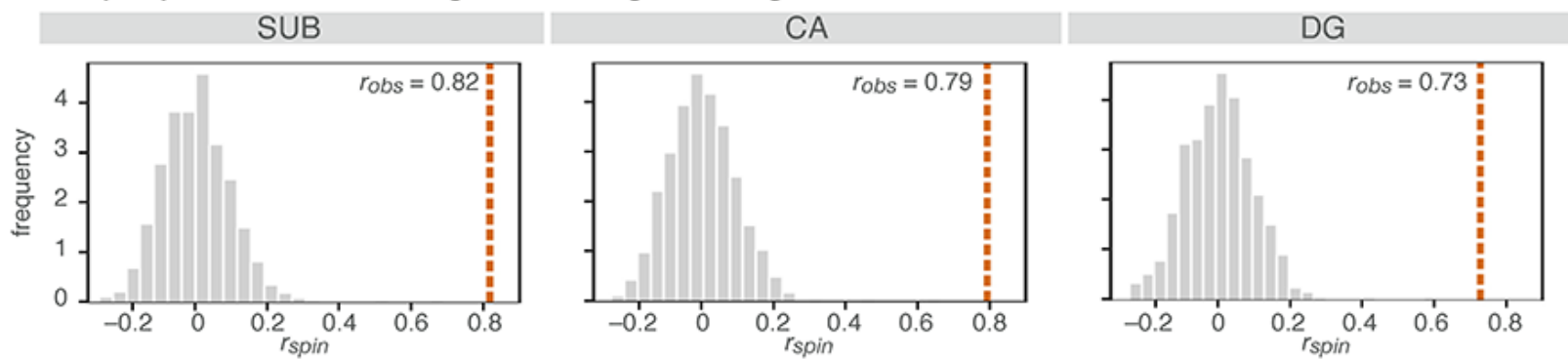

C Association between isocortical multiple demand axis and projections of primary fused gradient

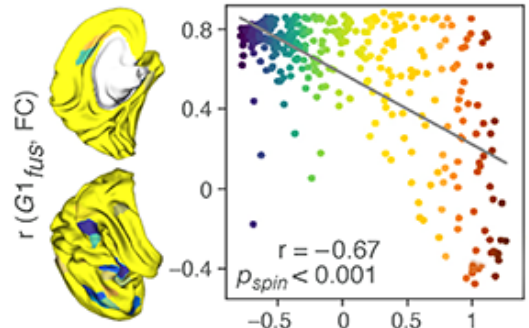

G3 (isocortex)

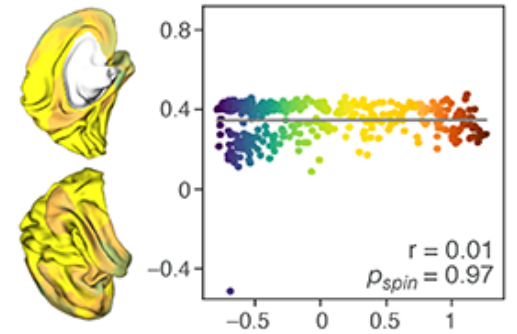

G3 (isocortex)

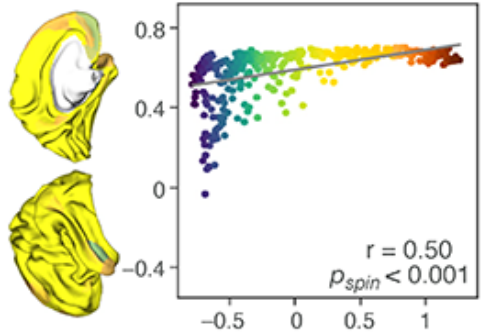

G3 (isocortex)

\section{Association between isocortical multiple demand axis and projections of secondary fused gradient}
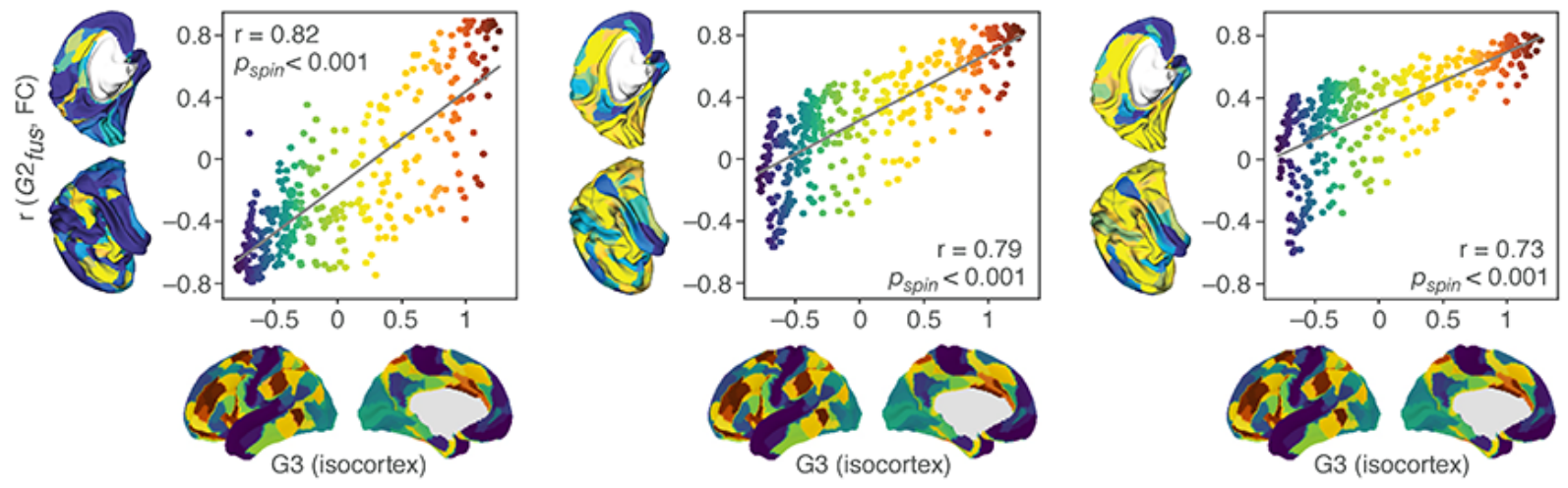
Supplementary Fig. S6. The association between the fused gradient projections and the third macroscale gradient. A. Gradient decomposition of whole-brain isocortical-isocortical functional connectivity and variance explained by the first 25 gradients. The principal gradient G1 (isocortex) reflects a sensory-transmodal axis, the second gradient G2 (isocortex) a visual-somatomotor axis, and the third gradient G3 (isocortex) the multiple demand axis versus sensorimotor and DMN networks. B. Exemplary demonstration for the significance level assessment by the spin permutations ${ }^{2,3}$ that controls the spatial auto-correlations. Observed correlations $r_{o b s}$ correspond to the Pearson correlations between $\mathrm{r}($ G2 $2_{\text {fus }}, \mathrm{FC}$ ) and G3 (isocortex) and spin correlations $r_{\text {spin }}$ correspond to that of the shuffled data by accounting for the surface coordinates $\left(n_{\text {perm }}=1000\right)$. C. Regional associations between the whole-brain multiple demand gradient (G3 (isocortex), x-axis) and the primary fused gradient projections on the isocortex $\left(\mathrm{r}\left(G 1_{f u s}, \mathrm{FC}\right), \mathrm{y}\right.$-axis). Associations were reported to be $\mathrm{r}=-0.67$ (Pearson) and $p_{\text {spin }}<0.001$ for SUB, $\mathrm{r}=0.01$ and $p_{\text {spin }}=0.97$ for $\mathrm{CA}$, and $\mathrm{r}=0.50$ and $p_{\text {spin }}<0.001$ for DG. D. Regional associations between the whole-brain multiple demand gradient (G3 (isocortex), x-axis) and the secondary fused gradient projections on the isocortex $\left(\mathrm{r}\left(G 2_{f u s}, \mathrm{FC}\right)\right.$, y-axis). Associations were quantified as $\mathrm{r}=0.82$ for $\mathrm{SUB}, \mathrm{r}=0.79$ for $\mathrm{CA}$, and $\mathrm{r}=0.73$ for $\mathrm{DG}$, and were all significantly reported by $p_{\text {spin }}<0.001$.

Supplementary Table S1. Pairwise correlations among the subfield organisational axes.

\begin{tabular}{|c|c|c|c|c|}
\hline$G 1_{f u s}$ & $\mathrm{r}=-0.29, p_{\text {vario }}=0.02$ & $\mathrm{r}=0.08, p_{\text {vario }}=0.48$ & $\mathrm{r}=0.46, p_{\text {vario }}=0.001$ & $\mathrm{r}=0.12, p_{\text {vario }}=0.30$ \\
\hline$G_{f u s}$ & $\mathrm{r}=0.89, p_{\text {vario }}<0.001$ & $\mathrm{r}=0.73, p_{\text {vario }}<0.001$ & $\mathrm{r}=0.12, p_{\text {vario }}=0.37$ & $\mathrm{r}=0.56, p_{\text {vario }}<0.001$ \\
\hline \multicolumn{5}{|l|}{ LCA } \\
\hline $\operatorname{G1}_{f u s}$ & $\mathrm{r}=0.29, p_{\text {vario }}=0.002$ & $\mathrm{r}=0.34, p_{\text {vario }}<0.001$ & $\mathrm{r}=0.14, p_{\text {vario }}=0.11$ & $\mathrm{r}=-0.14, p_{\text {vario }}=0.15$ \\
\hline$G 2_{f u s}$ & $\mathrm{r}=0.89, p_{\text {vario }}<0.001$ & $\mathrm{r}=0.83, p_{\text {vario }}<0.001$ & $\mathrm{r}=0.21, p_{\text {vario }}=0.04$ & $\mathrm{r}=0.10, p_{\text {vario }}=0.27$ \\
\hline$G 1_{f u s}$ & $\mathrm{r}=0.53, p_{\text {vario }}<0.001$ & $\mathrm{r}=0.41, p_{\text {vario }}=0.002$ & $\mathrm{r}=0.22, p_{\text {vario }}=0.03$ & $\mathrm{r}=0.00, p_{\text {vario }}=0.99$ \\
\hline $\mathrm{G}_{\text {fus }}$ & $\mathrm{r}=0.86, p_{\text {vario }}<0.001$ & $\mathrm{r}=0.80, p_{\text {vario }}<0.001$ & $\mathrm{r}=-0.26, p_{\text {vario }}=0.04$ & $\mathrm{r}=0.35, p_{\text {vario }}=0.02$ \\
\hline
\end{tabular}


Supplementary Table S2. The association between whole-brain functional gradients and the isocortical projections of fused gradients.

\begin{tabular}{|l|l|l|l|}
\hline LSUB & G1 (isocortex) & $\mathrm{G} 2$ (isocortex) & $\mathrm{G} 3$ (isocortex) \\
\hline $\mathrm{r}\left(G 1_{f u s}, \mathrm{FC}\right)$ & $\mathrm{r}=0.02, p_{\text {spin }}=0.81$ & $\mathrm{r}=-0.17, p_{\text {spin }}=0.31$ & $\mathrm{r}=-0.67, p_{\text {spin }}<0.001$ \\
\hline $\mathrm{r}\left(G 2_{f u s}, \mathrm{FC}\right)$ & $\mathrm{r}=-0.06, p_{\text {spin }}=0.60$ & $\mathrm{r}=-0.01, p_{\text {spin }}=0.97$ & $\mathrm{r}=0.82, p_{\text {spin }}<0.001$ \\
\hline $\mathbf{L C A}$ & $\mathrm{r}=-0.70, p_{\text {spin }}<0.001$ & $\mathrm{r}=0.04, p_{\text {spin }}=0.85$ & $\mathrm{r}=0.01, p_{\text {spin }}=0.97$ \\
\hline $\mathrm{r}\left(G 1_{f u s}, \mathrm{FC}\right)$ & $\mathrm{r}=-0.30, p_{\text {spin }}=0.002$ & $\mathrm{r}=-0.05, p_{\text {spin }}=0.73$ & $\mathrm{r}=0.79, p_{\text {spin }}<0.001$ \\
\hline $\mathrm{r}\left(G 2_{f u s}, \mathrm{FC}\right)$ & $\mathrm{r}=-0.54, p_{\text {spin }}<0.001$ & $\mathrm{r}=-0.08, p_{\text {spin }}=0.57$ & $\mathrm{r}=0.50, p_{\text {spin }}<0.001$ \\
\hline $\mathbf{L D G}$ & $\mathrm{r}=-0.48, p_{\text {spin }}<0.001$ & $\mathrm{r}=-0.03, p_{\text {spin }}=0.81$ & $\mathrm{r}=0.73, p_{\text {spin }}<0.001$ \\
\hline $\mathrm{r}\left(G 1_{f u s}, \mathrm{FC}\right)$ & $\mathrm{r}\left(G 2_{f u s}, \mathrm{FC}\right)$ &
\end{tabular}

\section{References}

1. Yeo, B. T. T. et al. The organization of the human cerebral cortex estimated by intrinsic functional connectivity. Journal of Neurophysiology vol. 106 1125-1165 (2011).

2. Vos de Wael, R. et al. BrainSpace: a toolbox for the analysis of macroscale gradients in neuroimaging and connectomics datasets. Commun Biol 3, 103 (2020).

3. Alexander-Bloch, A. F. et al. On testing for spatial correspondence between maps of human brain structure and function. Neuroimage 178, 540-551 (2018). 